\title{
Modeling and analysis of an elastic problem in large displacements and small strains
}

\author{
C. Grandmont, Y. Maday and P. Métier
}

7th April 2004

\begin{abstract}
In this paper we present and analyse a dynamical geometrically nonlinear formulation that models the motion of a two-dimensional elastic structure in large displacements-small strains. In a first part (section 2) we derive the equations describing the motion of the body. In a second part (section 3), existence of a weak solution is proven using a Galerkin method. We also prove that the solution is unique.
\end{abstract}

\section{Introduction}

In this paper, we present and analyse a dynamical geometrically nonlinear formulation that models the motion of a two-dimensional structure in large displacements-small strains. The starting point of the formulation is to seperate the rigid body motion from purely elastic displacements. This is an idea that has been used in the past two decades in engineering sciences and is referred in literature as the corotational kinematic description (see [6]).

In a first part, we derive the $2 \mathrm{D}$ model (section 2). We give a criterion that enables us to associated to deformations that are "close" to rigid body motion a unique translation, rotation and elastic displacement. This criterion was considered by de Veubeke in [7]. Writing the Lagrange equations of the problem, two equivalent weak formulations are obtained. We next obtain $a$ priori estimates for the solution. Unlike the linearized elasticity for which the rotations are not well described the model take into account the real rotations of the body and therefore is nonlinear. Nevertheless, this model is linear with respect to the elastic displacement, thus it can be seen as a model between the linearized elasticity and the standard system of elasticity for a Saint-Venant material for instance.

In the second part, we study the solvability of the problem. The proof of the existence of at least one local solution is based on a Galerkin method. We prove that the discrete problem has one solution bounded in the energy spaces. Because of the presence of nonlinear terms, additional bounds are needed in order to get compactness and obtain the continuous problem as the limit of the discrete one. These estimates are obtained by looking at the low and high frequencies of the acceleration separately. Then, we prove in two steps that the solution is unique. The first step consists in formal calculations and in a second part thoses calculations are justified using admissible test functions. Finally we prove the existence of a maximal solution. 


\section{Modeling}

\subsection{Decomposition of the deformation}

Let $\Omega$ be a lipchitz domain of $\mathbb{R}^{2}$. We suppose that $\bar{\Omega}$ is the reference configuration of an elastic body. We denote by $\phi: \bar{\Omega} \longrightarrow \mathbb{R}^{2}$ the deformation of this body: to each point $\xi \in \bar{\Omega}$ we associate the point $\mathbf{x}=\phi(\xi)$ of the deformed configuration $\phi(\bar{\Omega})$. The associated displacement is denoted by $\mathbf{u}=\phi-\mathrm{Id}_{\mathbb{R}^{2}}$ We want to obtain a model in order to describe the motion of this structure in large displacements and small strains. The key idea is to separate the displacements due to elastic behavior from the one due to rigid body motions. We thus consider that the deformation can be decomposed in

1. a translation whose vector is $\tau: \xi \longmapsto \xi+\tau$ with $\tau \in \mathbb{R}^{2}$.

2. a rotation : $\xi \longmapsto R_{\theta} \overrightarrow{G \xi}$, of angle $\theta$ and centre $G$, where $G$ denotes the centre of gravity of $\Omega$ and where $R_{\theta}=\left(\begin{array}{cc}\cos \theta & -\sin \theta \\ \sin \theta & \cos \theta\end{array}\right), \theta \in \mathbb{R}$.

3. an elastic deformation : $\bar{\Omega} \longrightarrow \mathbb{R}^{2}$

$$
\xi \longmapsto \xi+\mathbf{d}(\xi) \text {. }
$$

The deformation can be written as follows:

$$
\forall \xi \in \bar{\Omega}, \quad \phi(\xi)=\tau+R_{\theta}(\overrightarrow{G \xi}+\mathbf{d}(\xi))
$$

The rotation can be viewed as a rotation in $\mathbb{R}^{3}$ of angle $\theta$ and axis $\overrightarrow{e_{z}}$, where $\overrightarrow{e_{z}}$ denotes a unit vector orthogonal to the motion's plane $\mathbb{R}^{2}$

We first remark that for all $\phi$ in $L^{2}(\Omega)$, for all $\boldsymbol{\tau}$ in $\mathbb{R}^{2}$, for all $\theta$ in $\mathbb{R}, \mathbf{d}$ is defined uniquely thanks to (1) by $\mathbf{d}(\xi)=R_{\theta}^{T}(\phi(\xi)-\boldsymbol{\tau})-\overrightarrow{G \xi}$ and $\mathbf{d}$ belongs also to $L^{2}(\Omega)$.

The first step is to be able to associate a unique triplet $(\boldsymbol{\tau}, \theta, \mathbf{d})$ to a given $\phi$ in $L^{2}(\Omega)$. This will be possible for deformations $\phi$ "close" to rigid deformations in a sense that will be made precise latter on. The criterion we introduce is the minimization of the $L^{2}$-norm of $\mathbf{d}$. We thus consider the following mapping:

$$
\begin{aligned}
K_{\phi}: \mathbb{R}^{2} \times \mathbb{R} & \longrightarrow \mathbb{R} \\
(\boldsymbol{\tau}, \theta) & \longmapsto K_{\phi}(\boldsymbol{\tau}, \theta)=\left\|R_{\theta}^{T}(\phi-\boldsymbol{\tau})-\overrightarrow{G \xi}\right\|_{L^{2}(\Omega)}^{2}
\end{aligned}
$$

and we search the minimizer $(\tau, \theta)$ of this functional. Using the fact that $\int_{\Omega} \overrightarrow{G \xi}=0$ we have that

$$
K_{\phi}(\tau, \theta)=\|\phi-\tau\|^{2}-2 \int_{\Omega} \phi \cdot R_{\theta} \overrightarrow{G \xi}+\|\overrightarrow{G \xi}\|^{2} .
$$

We want to solve

$$
\min _{\substack{\boldsymbol{\theta} \in\left[0,2 \pi\left[ \\\tau \in \mathbb{R}^{2}\right.\right.}} K_{\phi}(\boldsymbol{\tau}, \theta)
$$

This problem has at least one solution since $K_{\phi}(\boldsymbol{\tau}, \theta)=k_{1}(\boldsymbol{\tau})+k_{2}(\theta)$, where $k_{1}$ is a coercive strictly convex mapping, and $k_{2}$ is continuous and $2 \pi$-periodic $\left(k_{1}(\boldsymbol{\tau})=\|\boldsymbol{\phi}-\boldsymbol{\tau}\|^{2}, k_{2}(\theta)=\right.$ $-2 \int_{\Omega} \phi \cdot R_{\theta} \overrightarrow{G \xi}+\|\overrightarrow{G \xi}\|^{2}$ ). The optimality conditions of order one and two are:

$$
\begin{array}{rlrl}
\frac{\partial K_{\phi}}{\partial \boldsymbol{\tau}}(\boldsymbol{\tau}, \theta) & =0, & \frac{\partial^{2} K_{\phi}}{\partial \boldsymbol{\tau}^{2}}(\boldsymbol{\tau}, \theta) & >0 \text { in the sense of symmetric matrix }, \\
\frac{\partial K_{\phi}}{\partial \theta}(\boldsymbol{\tau}, \theta) & =0, & \frac{\partial^{2} K_{\phi}}{\partial \theta^{2}}(\boldsymbol{\tau}, \theta) \geq 0 .
\end{array}
$$


They can be written in an equivalent way:

$$
\tau=\frac{1}{|\Omega|} \int_{\Omega} \phi, \quad \int_{\Omega} \phi \wedge R_{\theta} \overrightarrow{G \xi}=0, \quad \int_{\Omega} \phi \cdot R_{\theta} \overrightarrow{G \xi} \geq 0
$$

The first condition $\tau=\frac{1}{|\Omega|} \int_{\Omega} \phi$ determines uniquely $\tau$. The two other conditions can be rewritten as follows:

$$
\begin{aligned}
\int_{\Omega} \phi \wedge R_{\theta} \overrightarrow{G \xi}= & \left(\int_{\Omega} \phi \wedge \overrightarrow{G \xi}\right) \cos \theta+\left(\int_{\Omega} \phi \cdot \overrightarrow{G \xi}\right) \sin \theta=0 \\
\int_{\Omega} \phi \cdot R_{\theta} \overrightarrow{G \xi}= & -\left(\int_{\Omega} \phi \wedge \overrightarrow{G \xi}\right) \sin \theta+\left(\int_{\Omega} \phi \cdot \overrightarrow{G \xi}\right) \cos \theta \geq 0 .
\end{aligned}
$$


$(\boldsymbol{\tau}, \theta)$, we associate $\mathbf{d}$ defined by $\mathbf{d}(\xi)=R_{\theta}^{T}(\phi(\xi)-\boldsymbol{\tau})-\overrightarrow{G \xi}, \forall \xi \in \bar{\Omega}$. Next, taking into account the first condition of (3), and since $G$ is the center of gravity of $\Omega$, we remark that $\mathbf{d}$ has a zero average in $\Omega$. Moreover the conditions (4) can be rewritten as follows:

$$
\int_{\Omega} \phi \wedge R_{\theta} \overrightarrow{G \xi}=\int_{\Omega} \mathbf{d} \wedge \overrightarrow{G \xi}=0 \quad \text { and } \quad \int_{\Omega} \phi \cdot R_{\theta} \overrightarrow{G \xi}=\int_{\Omega}(\overrightarrow{G \xi}+\mathbf{d}) \cdot \overrightarrow{G \xi} \geq 0 .
$$

Remark that, taking into account the first condition of (4), if $\int_{\Omega} \phi \wedge \overrightarrow{G \xi} \neq 0$ or $\int_{\Omega} \phi \cdot \overrightarrow{G \xi} \neq 0$, then the second condition of (4) (or the second condition of (5)) is in fact a strict inequality.

Thus we have established the proposition:

Proposition 1 For all $\phi \in L^{2}(\Omega)$ such that:

$$
\left(\int_{\Omega} \phi \wedge \overrightarrow{G \xi}\right)^{2}+\left(\int_{\Omega} \phi \cdot \overrightarrow{G \xi}\right)^{2} \neq 0
$$

there exists a unique triplet $(\boldsymbol{\tau}, \theta, \mathbf{d}) \in \mathbb{R}^{2} \times\left[0,2 \pi\left[\times L^{2}(\Omega)\right.\right.$ satisfying $\boldsymbol{\tau}+R_{\theta}(\overrightarrow{G \xi}+\mathbf{d})=\boldsymbol{\phi}$ and such that d satisfies:

$$
\int_{\Omega} \mathbf{d}=0, \quad \int_{\Omega} \mathbf{d} \wedge \overrightarrow{G \xi}=0 \text { and } \int_{\Omega}(\mathbf{d}+\overrightarrow{G \xi}) \cdot \overrightarrow{G \xi}>0
$$

In particular $(\boldsymbol{\tau}, \theta)$ is the unique solution of $(\mathcal{P})$, and $\mathbf{d}=R_{\theta}^{T}(\phi-\tau)-\overrightarrow{G \xi}$.

In all what follows, we will suppose that $\phi$ satisfies (6). This assumption is justified since we are interested in deformations that are close to rigid deformations. Indeed, in the rigid case, we have $\mathbf{d} \equiv 0$ and consequently $\int_{\Omega} \phi \cdot R_{\theta} \overrightarrow{G \xi}=\int_{\Omega}|\overrightarrow{G \xi}|^{2}>0$.

Now that we have obtained a suitable representation of the deformations, let us make precise the sets we will use. Let $s$ be a positive real number. We set:

$$
\begin{aligned}
& \mathbf{X}_{s}=\left\{\boldsymbol{\phi} \in H^{s}(\Omega) ;\left(\int_{\Omega} \boldsymbol{\phi} \cdot \overrightarrow{G \xi}\right)^{2}+\left(\int_{\Omega} \phi \wedge \overrightarrow{G \xi}\right)^{2} \neq 0\right\}, \\
& \mathcal{E}_{s}=\left\{\mathbf{d} \in H^{s}(\Omega) ; \int_{\Omega} \mathbf{d}=0, \int_{\Omega} \mathbf{d} \wedge \overrightarrow{G \xi}=0\right\}, \\
& \mathbf{Y}_{s}=\left\{\mathbf{d} \in \mathcal{E}_{s} ; \int_{\Omega}(\overrightarrow{G \xi}+\mathbf{d}) \cdot \overrightarrow{G \xi}>0\right\}, \\
& \mathbf{Z}_{s}=\mathbb{R}^{2} \times\left[0,2 \pi\left[\times \mathbf{Y}_{s} .\right.\right.
\end{aligned}
$$


Here $H^{0}(\Omega)$ denotes the space $L^{2}(\Omega)$. One can note that $\mathcal{E}_{0}$ is a close subspace of $L^{2}(\Omega)$ whose vectors are orthogonal (for the scalar product of $L^{2}(\Omega)$ ) to the translations and infinitesimal rotations $\left(\xi \mapsto \overrightarrow{e_{z}} \wedge \overrightarrow{G \xi}\right)$. We now introduce the mapping

$$
\begin{aligned}
& \mathcal{H}: \quad \mathbb{R}^{2} \times \mathbb{R} \times L^{2}(\Omega) \quad \longrightarrow \quad L^{2}(\Omega) \\
& (\boldsymbol{\tau}, \theta, \mathbf{d}) \quad \longmapsto \phi=\boldsymbol{\tau}+R_{\theta}(\overrightarrow{G \xi}+\mathbf{d})
\end{aligned}
$$

The mapping satisfies the following proposition:

Proposition 2 The mapping $\mathcal{H}: \quad \mathbb{R}^{2} \times \mathbb{R} \times L^{2}(\Omega) \quad \longrightarrow \quad L^{2}(\Omega) \longrightarrow \quad$ is a $C^{1}$-diffeo-

$$
(\boldsymbol{\tau}, \theta, \mathbf{d}) \quad \longmapsto \phi=\boldsymbol{\tau}+R_{\theta}(\overrightarrow{G \xi}+\mathbf{d})
$$

morphisme from $\mathbf{Z}_{s}$ onto $\mathbf{X}_{s}$. For all $\phi \in \mathbf{X}_{s}$, the differential $D \mathcal{F}(\phi)$ of the inverse $\mathcal{F}$ of $\mathcal{H}$ is the linear mapping from $H^{s}(\Omega)$ onto $\mathbb{R}^{2} \times \mathbb{R} \times \mathcal{E}_{s}$ defined by $D \mathcal{F}(\phi) . \mathbf{v}=(\overline{\boldsymbol{\tau}}(\mathbf{v}), \bar{\theta}(\mathbf{v}), \overline{\mathbf{d}}(\mathbf{v}))$ where

$$
\begin{aligned}
& \overline{\boldsymbol{\tau}}(\mathbf{v})=\frac{1}{|\Omega|} \int_{\Omega} \mathbf{v} \\
& \bar{\theta}(\mathbf{v})=\frac{1}{\int_{\Omega}(\overrightarrow{G \xi}+\mathbf{d}) \cdot \overrightarrow{G \xi}} \int_{\Omega} \mathbf{v} \cdot\left(\overrightarrow{e_{z}} \wedge R_{\theta} \overrightarrow{G \xi}\right),
\end{aligned}
$$

and

$$
\overline{\mathbf{d}}(\mathbf{v})=R_{\theta}^{T}\left(\mathbf{v}-\frac{1}{|\Omega|} \int_{\Omega} \mathbf{v}-\frac{1}{\int_{\Omega}(\overrightarrow{G \xi}+\mathbf{d}) \cdot \overrightarrow{G \xi}}\left(\int_{\Omega} \mathbf{v} \cdot\left(\overrightarrow{e_{z}} \wedge R_{\theta} \overrightarrow{G \xi}\right)\right) \overrightarrow{e_{z}} \wedge R_{\theta}(\overrightarrow{G \xi}+\mathbf{d})\right),
$$

with $(\boldsymbol{\tau}, \theta, \mathbf{d})=\mathcal{F}(\phi)$.

Proof: We first prove the proposition for $s=0$. Using the previous definitions, Proposition 1 says that for all $\boldsymbol{\phi}$ in $\mathbf{X}_{0}$, there exists a unique triplet $(\boldsymbol{\tau}, \theta, \mathbf{d}) \in \mathbf{Z}_{0}$ such that: $\mathcal{H}(\boldsymbol{\tau}, \theta, \mathbf{d})=\boldsymbol{\phi}$. Furthermore, $\mathcal{H}\left(\mathbf{Z}_{0}\right) \subset \mathbf{X}_{0}$. Indeed for all $(\boldsymbol{\tau}, \theta, \mathbf{d}) \in \mathbf{Z}_{0}$, since:

$$
\int_{\Omega} \phi \wedge R_{\theta} \overrightarrow{G \xi}=\int_{\Omega} \mathbf{d} \wedge \overrightarrow{G \xi} \text { and } \quad \int_{\Omega} \phi \cdot R_{\theta} \overrightarrow{G \xi}=\int_{\Omega}(\overrightarrow{G \xi}+\mathbf{d}) \cdot \overrightarrow{G \xi}
$$

we have that

$$
\int_{\Omega} \phi \wedge R_{\theta} \overrightarrow{G \xi}=0 \quad \text { and } \quad \int_{\Omega} \phi \cdot R_{\theta} \overrightarrow{G \xi}>0
$$

hold. But if $\int_{\Omega} \phi \cdot R_{\theta} \overrightarrow{G \xi}$ (which is equal to $-\left(\int_{\Omega} \phi \wedge \overrightarrow{G \xi}\right) \sin \theta+\left(\int_{\Omega} \phi \cdot \overrightarrow{G \xi}\right) \cos \theta$ ) is strictly positive then $\phi \in \mathbf{X}_{0}$. Consequently, the mapping $\mathcal{H}$ is one to one from $\mathbf{Z}_{0}$ onto $\mathbf{X}_{0}$. We are going to show that this is a $C^{1}$-diffomorphism from $\mathbf{Z}_{0}$ onto $\mathbf{X}_{0}$ and study its differential together with the differential of $\mathcal{F}=\mathcal{H}^{-1}$.

The mapping $\mathcal{H}$ is of class $C^{\infty}$, and we have in particular:

$$
\forall(\overline{\boldsymbol{\tau}}, \bar{\theta}, \overline{\mathbf{d}}) \in \mathbb{R}^{2} \times \mathbb{R} \times \mathcal{E}_{0}, \quad D \mathcal{H}(\boldsymbol{\tau}, \theta, \mathbf{d}) \cdot(\overline{\boldsymbol{\tau}}, \bar{\theta}, \overline{\mathbf{d}})=\overline{\boldsymbol{\tau}}+\bar{\theta} \overrightarrow{e_{z}} \wedge R_{\theta}(\overrightarrow{G \xi}+\mathbf{d})+R_{\theta} \overline{\mathbf{d}}
$$

We want to prove that $\forall(\boldsymbol{\tau}, \theta, \mathbf{d}) \in \mathbf{Z}_{0}, \forall \mathbf{v} \in L^{2}(\Omega)$, there exists a unique triplet $(\overline{\boldsymbol{\tau}}(\mathbf{v}), \bar{\theta}(\mathbf{v}), \overline{\mathbf{d}}(\mathbf{v})) \in$ $\mathbb{R}^{2} \times \mathbb{R} \times \mathcal{E}_{0}$ such that $D \mathcal{H}(\boldsymbol{\tau}, \theta, \mathbf{d}) \cdot(\overline{\boldsymbol{\tau}}(\mathbf{v}), \bar{\theta}(\mathbf{v}), \overline{\mathbf{d}}(\mathbf{v}))=\mathbf{v}$. Thanks to the definition of $\mathcal{E}_{0}$ and $G$ we have the orthogonal decomposition of $L^{2}(\Omega)$ :

$$
L^{2}(\Omega)=\left\langle\overrightarrow{e_{x}}, \overrightarrow{e_{y}}\right\rangle \stackrel{\perp}{\oplus}\left\langle\overrightarrow{e_{z}} \wedge \overrightarrow{G \xi}\right\rangle \stackrel{\perp}{\oplus \mathcal{E}_{0}}
$$

this yields, for all $\theta \in \mathbb{R}$,

$$
L^{2}(\Omega)=\left\langle\overrightarrow{e_{x}}, \overrightarrow{e_{y}}\right\rangle \stackrel{\perp}{\oplus}\left\langle\overrightarrow{e_{z}} \wedge R_{\theta} \overrightarrow{G \xi}\right\rangle \stackrel{\perp}{\oplus}\left\langle R_{\theta} \overline{\mathbf{d}} ; \overline{\mathbf{d}} \in \mathcal{E}_{0}\right\rangle
$$


Considering $\mathbf{d} \in L^{2}(\Omega)$ such that $\int_{\Omega}(\overrightarrow{G \xi}+\mathbf{d}) \cdot \overrightarrow{G \xi} \neq 0$, we deduce from (11) that

$$
L^{2}(\Omega)=\left\langle\overrightarrow{e_{x}}, \overrightarrow{e_{y}}\right\rangle \oplus\left\langle\overrightarrow{e_{z}} \wedge R_{\theta}(\overrightarrow{G \xi}+\mathbf{d})\right\rangle \oplus\left\langle R_{\theta} \overline{\mathbf{d}} ; \overline{\mathbf{d}} \in \mathcal{E}_{0}\right\rangle
$$

This implies that for each $\mathbf{v} \in L^{2}(\Omega)$, there exists a unique triplet $(\overline{\boldsymbol{\tau}}(\mathbf{v}), \bar{\theta}(\mathbf{v}), \overline{\mathbf{d}}(\mathbf{v})) \in \mathbb{R}^{2} \times \mathbb{R} \times \mathcal{E}_{0}$ such that:

$$
\mathbf{v}=\overline{\boldsymbol{\tau}}(\mathbf{v})+\bar{\theta}(\mathbf{v}) \overrightarrow{e_{z}} \wedge R_{\theta}(\overrightarrow{G \xi}+\mathbf{d})+R_{\theta} \overline{\mathbf{d}}(\mathbf{v})
$$

Moreover we easily obtain

$$
\begin{aligned}
& \overline{\boldsymbol{\tau}}(\mathbf{v})=\frac{1}{|\Omega|} \int_{\Omega} \mathbf{v} \\
& \bar{\theta}(\mathbf{v})=\frac{1}{\int_{\Omega}(\overrightarrow{G \xi}+\mathbf{d}) \cdot \overrightarrow{G \xi}} \int_{\Omega} \mathbf{v} \cdot\left(\overrightarrow{e_{z}} \wedge R_{\theta} \overrightarrow{G \xi}\right)
\end{aligned}
$$

and thus

$$
\begin{aligned}
\overline{\mathbf{d}}(\mathbf{v}) & =R_{\theta}^{T}\left(\mathbf{v}-\overline{\boldsymbol{\tau}}(\mathbf{v})-\bar{\theta}(\mathbf{v}) \overrightarrow{e_{z}} \wedge R_{\theta}(\overrightarrow{G \xi}+\mathbf{d})\right) \\
& =R_{\theta}^{T}\left(\mathbf{v}-\frac{1}{|\Omega|} \int_{\Omega} \mathbf{v}-\frac{1}{\int_{\Omega}(\overrightarrow{G \xi}+\mathbf{d}) \cdot \overrightarrow{G \xi}}\left(\int_{\Omega} \mathbf{v} \cdot\left(\overrightarrow{e_{z}} \wedge R_{\theta} \overrightarrow{G \xi}\right)\right) \overrightarrow{e_{z}} \wedge R_{\theta}(\overrightarrow{G \xi}+\mathbf{d})\right) .
\end{aligned}
$$

Consequently for all $(\boldsymbol{\tau}, \theta, \mathbf{d}) \in \mathbf{Z}_{0}, D \mathcal{H}(\boldsymbol{\tau}, \theta, \mathbf{d})$ is an isomorphism from $\mathbb{R}^{2} \times \mathbb{R} \times \mathcal{E}_{0}$ onto $L^{2}(\Omega)$. And we have:

$$
D \mathcal{F}(\phi) . \mathbf{v}=(\overline{\boldsymbol{\tau}}(\mathbf{v}), \bar{\theta}(\mathbf{v}), \overline{\mathbf{d}}(\mathbf{v})),
$$

where $\phi \in \mathbf{X}_{0}$ and $\mathbf{v} \in L^{2}(\Omega)$.

For $s>0$, we see easily that if $\phi \in \mathbf{X}_{s}$ then $(\boldsymbol{\tau}, \theta, \mathbf{d}) \in \mathbf{Z}_{s}$ (and reciprocally). Moreover we deduce from (13) that if $\mathbf{v} \in H^{s}(\Omega)$ then $\overline{\mathbf{d}}(\mathbf{v}) \in \mathcal{E}_{s}$. Thus the mapping $\mathcal{F}$ is a $C^{1}$-diffeomorphism from $\mathbf{X}_{s}$ onto $\mathbf{Z}_{s}$, and the components of its differential at each $\phi \in \mathbf{X}_{s}$ are given by (8)-(9).

\subsection{Derivation of the model: weak formulation of the equations}

In this subsection, we derive a dynamical model that describes the motion of an homogeneous, isotropic, elastic body in large displacements, small strains. We assume that the reference configuration $\bar{\Omega}$ of the elastic media is a natural state. Under these hypotheses we can suppose that the material is a Saint Venant-Kirchhoff material. Its stored energy for a given displacement $\mathbf{u}$ is (cf for instance [2], Theorem 4.4.3 page 155):

$$
\check{W}(\mathbf{E}(\mathbf{u}))=\frac{\lambda}{2}[\operatorname{Tr}(\mathbf{E}(\mathbf{u}))]^{2}+\mu \operatorname{Tr}[\mathbf{E}(\mathbf{u})]^{2}
$$

where $\lambda$ and $\mu$ denote the Lamé constants of the material and $\mathbf{E}$ is the Green-Saint Venant strain tensor:

$$
\mathbf{E}(\mathbf{u})=\frac{1}{2}\left(\nabla \mathbf{u}+\nabla \mathbf{u}^{T}+\nabla \mathbf{u}^{T} \nabla \mathbf{u}\right) .
$$

An important remark is that, for a displacement $\mathbf{u}$ and the associated translation, angle of rotation and elastic displacement $(\boldsymbol{\tau}, \theta, \mathbf{d})$, we have $\mathbf{E}(\mathbf{u})=\mathbf{E}(\mathbf{d})$, which traduces that the strain tensor depends only on the elastic displacement and do not depend on rigid body motion. The stored energy thus appears as an invariant with respect to the rigid body motions. Assuming that $\nabla \mathbf{d}$ is small, the linearized versions of the strain tensor and of the stored energy function are:

$$
\begin{gathered}
\ddagger(\mathbf{d})=\frac{1}{2}\left(\nabla \mathbf{d}+(\nabla \mathbf{d})^{T}\right), \\
\hat{\mathcal{W}}(\mathbf{d})=\frac{\lambda}{2}[\operatorname{Tr}(\notin(\mathbf{d}))]^{2}+\mu \operatorname{Tr}[\notin(\mathbf{d})]^{2} .
\end{gathered}
$$


We would like to describe the dynamical behavior of such a material under the hypothesis that $\mathbf{d}$ is "small" (in a sense to precise). Let $T$ be a non negative real number. For any $t \in[0, T]$, the deformation $\phi(\cdot, t)$ is decomposed at each time $t$ according to (1):

$$
\forall \xi \in \bar{\Omega}, \quad \phi(\xi, t)=\boldsymbol{\tau}(t)+R_{\theta(t)}(\overrightarrow{G \xi}+\mathbf{d}(\xi, t)) .
$$

We write the Lagrange equations satisfied by $\mathbf{u}=\phi-\mathrm{Id}_{\mathbb{R}^{2}}$ (one can refer to [1] or [4] for details on Lagrange equations). We denote by $\dot{\mathbf{u}}=\partial_{t} \mathbf{u}$ the body velocity, and we choose $\mathbf{u}$ (respectivelly $\dot{\mathbf{u}}$ ) as the generalized coordinate (resp. velocity). We introduce the external work function:

$$
T(\mathbf{u}, \dot{\mathbf{u}})=\int_{\Omega} \mathbf{f} \cdot \mathbf{u}+\int_{\partial \Omega} \mathbf{g} \cdot \mathbf{u},
$$

where $\mathbf{f}$ denotes the applied body force and $\mathbf{g}$ the applied surface force. For the elastic displacement $\mathbf{d}$, we define the stored energy function $\hat{\mathcal{W}}$ by (14), and the strain energy associated to $\mathbf{u}$ by:

$$
W(\mathbf{u}, \dot{\mathbf{u}})=\mathcal{W}(\mathbf{d})=\int_{\Omega} \hat{\mathcal{W}}(\mathbf{d})
$$

Finally the kinetic energy is denoted by $E_{C}=\rho_{S} \int_{\Omega}(\dot{\mathbf{u}})^{2} / 2$ where $\rho_{S}$ is the body density. The lagrangian of the stucture is then $\mathcal{L}(\mathbf{u}, \dot{\mathbf{u}})=E_{C}+T-W$, and the Lagrange equations of the system read as follows

$$
\begin{gathered}
\forall T>0, \forall \mathbf{v}, \mathbf{v}(\cdot, t=0)=\mathbf{v}(\cdot, t=T)=\partial_{t} \mathbf{v}(\cdot, t=0)=\partial_{t} \mathbf{v}(\cdot, t=T) \equiv 0, \\
\int_{0}^{T}\left(\frac{\partial \mathcal{L}}{\partial \dot{\mathbf{u}}}(\mathbf{u}, \dot{\mathbf{u}}) \cdot \partial_{t} \mathbf{v}+\frac{\partial \mathcal{L}}{\partial \mathbf{u}}(\mathbf{u}, \dot{\mathbf{u}}) \cdot \mathbf{v}\right)=0 .
\end{gathered}
$$

For all $\mathbf{u}, \dot{\mathbf{u}}, \mathbf{v}$ and $\dot{\mathbf{v}}$, we can see easily that

$$
\begin{array}{llll}
\frac{\partial E_{C}}{\partial \mathbf{u}}(\mathbf{u}, \dot{\mathbf{u}}) \cdot \mathbf{v} & =0, & \frac{\partial E_{C}}{\partial \dot{\mathbf{u}}}(\mathbf{u}, \dot{\mathbf{u}}) \cdot \dot{\mathbf{v}} & =\rho_{S} \\
\frac{\partial T}{\partial \mathbf{u}}(\mathbf{u}, \dot{\mathbf{u}}) \cdot \mathbf{v} & =\int_{\Omega} \mathbf{f} \cdot \mathbf{v}+\int_{\partial \Omega} \mathbf{g} \cdot \mathbf{v}, & \frac{\partial T}{\partial \dot{\mathbf{u}}}(\mathbf{u}, \dot{\mathbf{u}}) \cdot \dot{\mathbf{v}} & =0, \\
& \frac{\partial W}{\partial \dot{\mathbf{u}}}(\mathbf{u}, \dot{\mathbf{u}}) \cdot \dot{\mathbf{v}} & =0 .
\end{array}
$$

Recalling that $\mathbf{u}=\phi-\mathrm{Id}_{\mathbb{R}^{2}}$, using the fact that $D_{\mathbf{u}} \phi(\mathbf{u})=\mathrm{Id}$ and Proposition 2 we obtain:

$$
\forall \mathbf{v}, \quad \frac{\partial W}{\partial \mathbf{u}}(\mathbf{u}, \dot{\mathbf{u}}) \cdot \mathbf{v}=\int_{\Omega} D_{\mathbf{d}} \hat{\mathcal{W}}(\mathbf{d}) \cdot\left[D_{\phi} \mathbf{d}(\phi) \cdot\left(D_{\mathbf{u}} \phi(\mathbf{u}) \cdot \mathbf{v}\right)\right]=\int_{\Omega} D_{\mathbf{d}} \hat{\mathcal{W}}(\mathbf{d}) \cdot \overline{\mathbf{d}}
$$

where $\overline{\mathbf{d}}$ is associated to $\mathbf{v}$ through (9). Moreover

$$
D_{\mathbf{d}} \hat{\mathcal{W}}(\mathbf{d}) \cdot \mathbf{b}=\lambda \operatorname{Tr}(\notin(\mathbf{d})) \operatorname{Tr}(\notin(\mathbf{b}))+2 \mu \operatorname{Tr}[\notin(\mathbf{d}) \notin(\mathbf{b})],
$$

where $I_{2}$ is the identy matrix of $\mathbb{R}^{2}$. Using the notation $A: B=\operatorname{Tr}\left(B^{T} A\right), \forall A, B \in \mathcal{M}_{2}(\mathbb{R})$ this leads to,

$$
\frac{\partial W}{\partial \mathbf{u}}(\mathbf{u}, \dot{\mathbf{u}}) \cdot \mathbf{v}=\int_{\Omega} \bar{\sigma}(\mathbf{d}): \notin(\overline{\mathbf{d}}),
$$

with $\bar{\sigma}(\mathbf{d})=\lambda \operatorname{Tr}(\$(\mathbf{d})) I_{2}+2 \mu \notin(\mathbf{d})$. Finally, for $\mathbf{v}$ sufficiently smooth such as $\mathbf{v}(\cdot, 0)=\mathbf{v}(\cdot, T) \equiv 0$ and $\partial_{t} \mathbf{v}(\cdot, 0)=\partial_{t} \mathbf{v}(\cdot, T) \equiv 0$, we have:

$$
\int_{0}^{T}\left(\rho_{S} \int_{\Omega} \partial_{t} \mathbf{u} \cdot \partial_{t} \mathbf{v}+\int_{\Omega} \mathbf{f} \cdot \mathbf{v}+\int_{\partial \Omega} \mathbf{g} \cdot \mathbf{v}-\int_{\Omega} \bar{\sigma}(\mathbf{d}): \notin(\overline{\mathbf{d}})\right)=0
$$


with $\overline{\mathbf{d}}$ associated to $\mathbf{v}$ through (9). From the previous equation we deduce the weak formulation of the equations satisfied by $\mathbf{u}$ and by the associated elastic displacement $\mathbf{d}$ :

$\left\{\begin{array}{l}\forall \mathbf{v} \text { sufficiently smooth and } \overline{\mathbf{d}} \text { associated to } \mathbf{v} \text { through }(9), \\ \rho_{S} \int_{\Omega}\left(\partial_{t t} \mathbf{u}\right) \cdot \mathbf{v}+\int_{\Omega} \bar{\sigma}(\mathbf{d}): \notin(\overline{\mathbf{d}})=\int_{\Omega} \mathbf{f} \cdot \mathbf{v}+\int_{\partial \Omega} \mathbf{g} \cdot \mathbf{v} \quad \text { in the sense of distributions } \mathcal{D}^{\prime}(0, T) .\end{array}\right.$

This system has to be completed by initial data: $\mathbf{u}(\cdot, t=0)=\mathbf{u}_{0}$ and $\partial_{t} \mathbf{u}(\cdot, t=0)=\mathbf{u}_{1}$.

We have obtained equations that model the motion of a structure with large displacements and small strains, that are valid for elastic displacements $\mathbf{d}$ satisfying $\int_{\Omega}(\overrightarrow{G \xi}+\mathbf{d}) \cdot \overrightarrow{G \xi}>0$ (or at least $\left.\int_{\Omega}(\overrightarrow{G \xi}+\mathbf{d}) \cdot \overrightarrow{G \xi} \neq 0\right)$. This model belongs to the class of the so called geometrically nonlinear models. The weak formulation we obtain is not surprising since we have a term of acceleration depending on the global displacement and a term of mechanical energy depending only on the elastic displacement. Note however that this formulation is not standard since the test functions depend on the solution.

Remark 1 From this weak formulation, a strong formulation can be deduced at least formally. Denoting by $\mathcal{P}_{\mathbf{u}}: H^{1}(\Omega) \longrightarrow H^{1}(\Omega)$ the linear operator which to any $\mathbf{v}$ associates the vector $\overline{\mathbf{d}}$ defined by (9), the weak formulation (17), becomes:

$$
\begin{aligned}
\int_{\Omega} \mathbf{f} \cdot \mathbf{v}+\int_{\partial \Omega} \mathbf{g} \cdot \mathbf{v}-\rho_{S} \int_{\Omega}\left(\partial_{t t} \mathbf{u}\right) \cdot \mathbf{v} & =\int_{\Omega} \bar{\sigma}(\mathbf{d}): \nabla\left(\mathcal{P}_{\mathbf{u}}(\mathbf{v})\right) \\
& =-\int_{\Omega} \mathcal{P}_{\mathbf{u}}^{T}[\operatorname{div}(\bar{\sigma}(\mathbf{d}))] \cdot \mathbf{v}+\int_{\partial \Omega}\left[\mathcal{P}_{\mathbf{u}}^{T}(\bar{\sigma}(\mathbf{d}) \cdot \mathbf{n})\right] \cdot \mathbf{v}
\end{aligned}
$$

Consequently for $\mathbf{v}$ smooth enough, this leads to the following equations

$$
\left\{\begin{array}{rlll}
\rho_{S} \partial_{t t} \mathbf{u}-\mathcal{P}_{\mathbf{u}}^{T}[\operatorname{div}(\bar{\sigma}(\mathbf{d}))] & =\mathbf{f} & & \text { in } \Omega \times(0, T) \\
\mathcal{P}_{\mathbf{u}}^{T}(\bar{\sigma}(\mathbf{d}) \cdot \mathbf{n}) & =\mathbf{g} & & \text { on } \partial \Omega \times(0, T) .
\end{array}\right.
$$

Those equations are nonlinear since the operator $\mathcal{P}_{\mathbf{u}}$ depends on the solution.

\subsection{Dynamic of the translation, rotation and elastic displacement}

In this subsection we derive a set of three equations equivalent to (17), these equations describe the dynamic of the translation $\boldsymbol{\tau}$, the angle of rotation $\theta$ and the elastic displacement $\mathbf{d}$. These weak formulations are obtained by taking appropriate test functions in (17).

For a given $\theta \in \mathbb{R}$ and a given $\mathbf{d} \in \mathbf{Y}_{1}$, we remark that, as what was done to obtain (12), $H^{1}(\Omega)$ is the sum of three subspaces:

$$
H^{1}(\Omega)=\left\langle\overrightarrow{e_{x}}, \overrightarrow{e_{y}}\right\rangle \oplus\left\langle\overrightarrow{e_{z}} \wedge R_{\theta}(\overrightarrow{G \xi}+\mathbf{d})\right\rangle \oplus\left\langle R_{\theta} \overline{\mathbf{d}} ; \overline{\mathbf{d}} \in \mathcal{E}_{1}\right\rangle
$$

Thus we will choose the test fonction $\mathbf{v}$ in (17), succesively belonging to $<\overrightarrow{e_{x}}, \overrightarrow{e_{y}}>,<\overrightarrow{e_{z}} \wedge(\overrightarrow{G \xi}+$ d) $>$ then equal to $R_{\theta} \overline{\mathbf{d}}$ where $\overline{\mathbf{d}} \in \mathcal{E}_{1}$ :

- If the mass of the structure is denoted by $m, m=\rho_{S}|\Omega|$, we obtain for $\mathbf{v} \in<\overrightarrow{e_{x}}, \overrightarrow{e_{y}}>$ :

$$
m \ddot{\boldsymbol{\tau}}=\rho_{S} \int_{\Omega} \partial_{t t} \mathbf{u}=\int_{\Omega} \mathbf{f}+\int_{\partial \Omega} \mathbf{g}
$$

since $\mathbf{d}$ has a zero average and $\mathbf{u}=\boldsymbol{\tau}+R_{\theta}(\overrightarrow{G \xi}+\mathbf{d})-\overrightarrow{O \xi}$. 
- Choosing $\overrightarrow{e_{z}} \wedge R_{\theta}(\overrightarrow{G \xi}+\mathbf{d})$ as a test function in (17), we obtain:

$$
\rho_{S} \int_{\Omega} R_{\theta}(\overrightarrow{G \xi}+\mathbf{d}) \wedge \partial_{t t} \mathbf{u}=\int_{\Omega} R_{\theta}(\overrightarrow{G \xi}+\mathbf{d}) \wedge \mathbf{f}+\int_{\partial \Omega} R_{\theta}(\overrightarrow{G \xi}+\mathbf{d}) \wedge \mathbf{g}
$$

Here the exterior product has to be taken as an operator defined on $\mathbb{R}^{2}$. In all what follows the exterior product $\wedge$ stands for both the exterior product of $\mathbb{R}^{3}$ and the operator defined on $\mathbb{R}^{2}$. We next express $\mathbf{u}$ with respect to $\boldsymbol{\tau}, \theta$ and $\mathbf{d}$ and differentiate twice:

$$
\partial_{t t} \mathbf{u}-\ddot{\boldsymbol{\tau}}=\ddot{R_{\theta}}(\overrightarrow{G \xi}+\mathbf{d})+2 \dot{R}_{\theta} \partial_{t} \mathbf{d}+R_{\theta} \partial_{t t} \mathbf{d} .
$$

Thus this gives a first contribution:

$$
\begin{gathered}
\int_{\Omega} R_{\theta}(\overrightarrow{G \xi}+\mathbf{d}) \wedge \ddot{R}_{\theta}(\overrightarrow{G \xi}+\mathbf{d})=\int_{\Omega} R_{\theta}(\overrightarrow{G \xi}+\mathbf{d}) \wedge\left[\ddot{\theta} \overrightarrow{e_{z}} \wedge R_{\theta}(\overrightarrow{G \xi}+\mathbf{d})+\dot{\theta} \overrightarrow{e_{z}} \wedge\left(\dot{\theta} \overrightarrow{e_{z}} \wedge R_{\theta}(\overrightarrow{G \xi}+\mathbf{d})\right)\right] \\
=\ddot{\theta} \frac{J(t)}{\rho_{S}}+(\dot{\theta})^{2} R_{\theta} \int_{\Omega}(\overrightarrow{G \xi}+\mathbf{d}) \wedge\left(\overrightarrow{e_{z}} \wedge\left(\overrightarrow{e_{z}} \wedge(\overrightarrow{G \xi}+\mathbf{d})\right)\right)=\ddot{\theta} \frac{J(t)}{\rho_{S}}
\end{gathered}
$$

where $J(t)=\rho_{S} \int_{\Omega}(\overrightarrow{G \xi}+\mathbf{d})^{2}$ denotes the inertia momemtum at time $t$, and where we have used the fact that $\dot{R}_{\theta} .=\dot{\theta} \overrightarrow{e_{z}} \wedge R_{\theta}$. and that $\overrightarrow{G \xi}+\mathbf{d}$ and $\overrightarrow{e_{z}} \wedge\left(\overrightarrow{e_{z}} \wedge(\overrightarrow{G \xi}+\mathbf{d})\right)$ are colinear. The second contribution can be written

$$
\begin{aligned}
\int_{\Omega} R_{\theta}(\overrightarrow{G \xi}+\mathbf{d}) \wedge \dot{R}_{\theta}\left(\partial_{t} \mathbf{d}\right) & =\dot{\theta} R_{\theta} \int_{\Omega}(\overrightarrow{G \xi}+\mathbf{d}) \wedge\left(\overrightarrow{e_{z}} \wedge \partial_{t} \mathbf{d}\right)=\dot{\theta}\left(\int_{\Omega}(\overrightarrow{G \xi}+\mathbf{d}) \cdot \partial_{t} \mathbf{d}\right) \\
& =\frac{\dot{\theta}}{2} \frac{d}{d t}\left(\frac{J(t)}{\rho_{S}}\right) .
\end{aligned}
$$

Using (22) together with the two previous equalities and using the fact that $\boldsymbol{\tau}$ is orthogonal to $\overrightarrow{e_{z}} \wedge R_{\theta}(\overrightarrow{G \xi}+\mathbf{d})$ yields:

$$
\rho_{S} \int_{\Omega} R_{\theta}(\overrightarrow{G \xi}+\mathbf{d}) \wedge \partial_{t t} \mathbf{u}=\frac{d}{d t}(\dot{\theta} J)+\rho_{S} \int_{\Omega}(\overrightarrow{G \xi}+\mathbf{d}) \wedge \partial_{t t} \mathbf{d}
$$

Moreover since $\mathbf{d}$ is orthogonal to the infinitesimal rotation $\xi \mapsto \overrightarrow{e_{z}} \wedge \overrightarrow{G \xi}$ we have,

$$
\rho_{S} \int_{\Omega} R_{\theta}(\overrightarrow{G \xi}+\mathbf{d}) \wedge \partial_{t t} \mathbf{u}=\frac{d}{d t}(\dot{\theta} J)+\rho_{S} \int_{\Omega} \mathbf{d} \wedge \partial_{t t} \mathbf{d}
$$

Then (21) becomes:

$$
\frac{d}{d t}(\dot{\theta} J)+\rho_{S} \int_{\Omega} \mathbf{d} \wedge \partial_{t t} \mathbf{d}=\int_{\Omega} R_{\theta}(\overrightarrow{G \xi}+\mathbf{d}) \wedge \mathbf{f}+\int_{\partial \Omega} R_{\theta}(\overrightarrow{G \xi}+\mathbf{d}) \wedge \mathbf{g} .
$$

- Finally to derive the equations satisfied by $\mathbf{d}$, we choose $\mathbf{v}=R_{\theta} \overline{\mathbf{d}}$ as a test function in (17), with $\overline{\mathbf{d}} \in \mathcal{E}_{1}$ :

$$
\rho_{S} \int_{\Omega} \partial_{t t} \mathbf{u} \cdot R_{\theta} \overline{\mathbf{d}}+\int_{\Omega} \bar{\sigma}(\mathbf{d}): \notin(\overline{\mathbf{d}})=\int_{\Omega} \mathbf{f} \cdot R_{\theta} \overline{\mathbf{d}}+\int_{\partial \Omega} \mathbf{g} \cdot R_{\theta} \overline{\mathbf{d}}
$$

Once again using (22) and the fact that $\overline{\mathbf{d}}$ is orthogonal to the translations:

$$
\int_{\Omega} \partial_{t t} \mathbf{u} \cdot R_{\theta} \overline{\mathbf{d}}=\int_{\Omega}\left(\ddot{R}_{\theta}(\overrightarrow{G \xi}+\mathbf{d})+2 \dot{R}_{\theta} \partial_{t} \mathbf{d}+R_{\theta} \partial_{t t} \mathbf{d}\right) \cdot R_{\theta} \overline{\mathbf{d}} .
$$


Note we have also:

$$
\begin{aligned}
\int_{\Omega} \ddot{R_{\theta}}(\overrightarrow{G \xi}+\mathbf{d}) \cdot R_{\theta} \overline{\mathbf{d}} & =\int_{\Omega}\left[\ddot{\theta} \overrightarrow{e_{z}} \wedge(\overrightarrow{G \xi}+\mathbf{d})+\dot{\theta} \overrightarrow{e_{z}} \wedge\left(\dot{\theta} \overrightarrow{e_{z}} \wedge(\overrightarrow{G \xi}+\mathbf{d})\right)\right] \cdot \mathbf{d} \\
& =\ddot{\theta} \overrightarrow{e_{z}} \cdot \int_{\Omega} \mathbf{d} \wedge \overline{\mathbf{d}}-(\dot{\theta})^{2} \int_{\Omega}(\overrightarrow{G \xi}+\mathbf{d}) \cdot \overline{\mathbf{d}}
\end{aligned}
$$

since $\overline{\mathbf{d}}$ again is orthogonal to the infinitesimal rotation. We also have:

$$
\int_{\Omega} \dot{R}_{\theta} \partial_{t} \mathbf{d} \cdot R_{\theta} \overline{\mathbf{d}}=\dot{\theta} \int_{\Omega}\left(\overrightarrow{e_{z}} \wedge \partial_{t} \mathbf{d}\right) \cdot \overline{\mathbf{d}}=\dot{\theta}\left(\int_{\Omega} \partial_{t} \mathbf{d} \wedge \overline{\mathbf{d}}\right)
$$

The previous equalities and (24) leads to:

$$
\begin{aligned}
\forall \overline{\mathbf{d}} \in \mathcal{E}_{1}, \quad \rho_{S}\left(\int_{\Omega} \partial_{t t} \mathbf{d} \cdot \overline{\mathbf{d}}+2 \dot{\theta} \int_{\Omega} \partial_{t} \mathbf{d} \wedge \overline{\mathbf{d}}+\ddot{\theta} \int_{\Omega} \mathbf{d} \wedge \overline{\mathbf{d}}-(\dot{\theta})^{2} \int_{\Omega}(\overrightarrow{G \xi}+\mathbf{d}) \cdot \overline{\mathbf{d}}\right) \\
+\int_{\Omega} \bar{\sigma}(\mathbf{d}): \nabla \overline{\mathbf{d}}=\int_{\Omega} \mathbf{f} \cdot R_{\theta} \overline{\mathbf{d}}+\int_{\partial \Omega} \mathbf{g} \cdot R_{\theta} \overline{\mathbf{d}} .
\end{aligned}
$$

These equations (20)-(23)-(25) have to be completed by initial data:

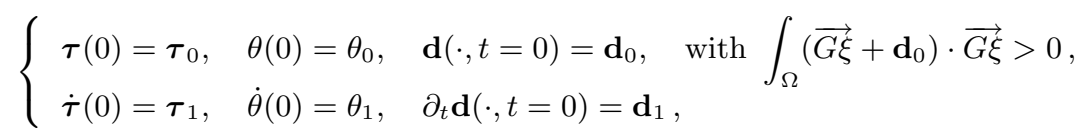

where:

$$
\left\{\begin{aligned}
\boldsymbol{\tau}_{0}+R_{\theta_{0}}\left(\overrightarrow{G \xi}+\mathbf{d}_{0}\right)-\overrightarrow{O \xi} & :=\mathbf{u}_{0}, \\
\boldsymbol{\tau}_{1}+R_{\theta_{0}} \mathbf{d}_{1}+\theta_{1} \overrightarrow{e_{z}} \wedge R_{\theta_{0}}\left(\overrightarrow{G \xi}+\mathbf{d}_{0}\right) & :=\mathbf{u}_{1}
\end{aligned}\right.
$$

Remark 2 The weak formulation (25) corresponds to the weak formulation of the equations that describe the motion of a linearized elastic body in rotation at velocity $\dot{\theta}$, equations written in the moving frame where Coriolis and centrifugal terms appear.

Remark 3 The equations (20), (23), (25) can be also obtained by using the Lagrangian with $q_{1}=$ $\boldsymbol{\tau}, q_{2}=\theta, q_{3}=\mathbf{d}$ as generalized coordinates. Thus $\mathcal{L}=\mathcal{L}\left(q_{1}, q_{2}, q_{3}, \dot{q}_{1}, \dot{q}_{2}, \dot{q}_{3}\right)$ and $\frac{d}{d t}\left(\frac{\partial \mathcal{L}}{\partial \dot{q}_{i}}\right)-$ $\frac{\partial \mathcal{L}}{\partial q_{i}}=0$ for $i=1,2,3$ give (20), (23) and (25).

\subsection{A priori estimates}

In the present subsection we derive a priori estimates assuming that there exists a solution $\mathbf{u}$ of (17) smooth enough. In a first step we establish an energy equality then energy estimates for the global system and in a second step we find bounds on the different components $(\boldsymbol{\tau}, \theta, \mathbf{d})$ of the displacement $\mathbf{u}$.

We take $\mathbf{v}=\partial_{t} \mathbf{u}=\dot{\boldsymbol{\tau}}+\dot{\theta} \overrightarrow{e_{z}} \wedge R_{\theta}(\overrightarrow{G \xi}+\mathbf{d})+R_{\theta} \partial_{t} \mathbf{d}$ as a test function in (17), which is equivalent to multiply (20) by $\dot{\boldsymbol{\tau}},(23)$ by $\dot{\theta}$, take $\partial_{t} \mathbf{d}$ as a test function in (25) and add these three contributions. The first term gives the derivative with respect to time of the structure kinetic energy. Moreover, setting

$$
\begin{aligned}
((\mathbf{d}, \mathbf{b})) & =\int_{\Omega}(\lambda \operatorname{Tr}(\notin(\mathbf{d})) \operatorname{Tr}(\$(\mathbf{b}))+2 \mu \notin(\mathbf{d}): \notin(\mathbf{b})), \\
\text { and } \quad\|\mathbf{b}\| \|^{2} & =((\mathbf{b}, \mathbf{b})),
\end{aligned}
$$

and observing that $\left\||\mathbf{d} \||^{2}=2 \mathcal{W}(\mathbf{d})\right.$, we obtain the following energy equality:

$$
\frac{\rho_{S}}{2} \frac{d}{d t}\left(\int_{\Omega}\left|\partial_{t} \mathbf{u}\right|^{2}\right)+\frac{d}{d t}[\mathcal{W}(\mathbf{d})]=\int_{\Omega} \mathbf{f} \cdot \partial_{t} \mathbf{u}+\int_{\partial \Omega} \mathbf{g} \cdot \partial_{t} \mathbf{u}
$$


Let us now establish a priori estimates. For $t \in(0, T)$, we write:

$$
\int_{\partial \Omega} \mathbf{g} \cdot \partial_{t} \mathbf{u}=\frac{d}{d t}\left(\int_{\partial \Omega} \mathbf{g} \cdot \mathbf{u}\right)-\int_{\partial \Omega} \partial_{t} \mathbf{g} \cdot \mathbf{u}
$$

and we denote by $E(t)$ the structure energy:

$$
E(t)=\frac{1}{2} \rho_{S}\left\|\partial_{t} \mathbf{u}(t)\right\|_{L^{2}(\Omega)}^{2}+\frac{1}{2}\|\mathbf{d}(t)\| \|^{2} .
$$

Then, integrating $(26)$ over $(0, t)$, we have:

$$
E(t)=E(0)-\int_{\partial \Omega} \mathbf{g}(0) \cdot \mathbf{u}(0)+\int_{0}^{t} \int_{\Omega} \mathbf{f}(s) \cdot \partial_{t} \mathbf{u}(s) \mathrm{d} s+\int_{\partial \Omega} \mathbf{g}(t) \cdot \mathbf{u}(t)-\int_{0}^{t} \int_{\partial \Omega} \partial_{t} \mathbf{g}(s) \cdot \mathbf{u}(s) \mathrm{d} s
$$

This leads to the following estimate:

$$
\begin{array}{r}
E(t) \leq a_{0}+\left(\frac{1}{2 \rho_{S}}\|\mathbf{f}\|_{L^{2}\left(0, T ; L^{2}(\Omega)\right)}^{2}+\int_{0}^{t} E(s) \mathrm{d} s\right)+C\|\mathbf{g}(t)\|_{H^{-1 / 2}(\partial \Omega)}\|\mathbf{u}(t)\|_{H^{1}(\Omega)} \\
+C \int_{0}^{t}\left\|\partial_{t} \mathbf{g}(s)\right\|_{H^{-1 / 2}(\partial \Omega)}\|\mathbf{u}(s)\|_{H^{1}(\Omega)} d s
\end{array}
$$

with $a_{0}=E(0)+\left|\int_{\partial \Omega} \mathbf{g}(0) \cdot \mathbf{u}_{0}\right|$ and where we used that for any $\mathbf{b} \in H^{1}(\Omega)$ :

$$
\|\mathbf{b}\|_{H^{1 / 2}(\partial \Omega)} \leq C\|\mathbf{b}\|_{H^{1}(\Omega)} .
$$

Next we estimate $\|\mathbf{u}(t)\|_{H^{1}(\Omega)}$ with respect to $E(t)$. Remembering that $\mathbf{u}=\boldsymbol{\tau}+R_{\theta}(\overrightarrow{G \xi}+\mathbf{d})-\overrightarrow{O \xi}$, it gives :

$$
\|\mathbf{u}(t)\|_{H^{1}(\Omega)}^{2} \leq 3\left(\|\boldsymbol{\tau}(t)\|_{H^{1}(\Omega)}^{2}+\left\|R_{\theta} \overrightarrow{G \xi}-\overrightarrow{O \xi}\right\|_{H^{1}(\Omega)}^{2}+\|\mathbf{d}(t)\|_{H^{1}(\Omega)}^{2}\right) .
$$

It is clear that:

$$
\left\|R_{\theta} \overrightarrow{G \xi}-\overrightarrow{O \xi}\right\|_{H^{1}(\Omega)}^{2} \leq C
$$

where the constant $C$ depends on $\Omega$, and that:

$$
\begin{aligned}
\|\boldsymbol{\tau}(t)\|_{H^{1}(\Omega)}^{2} & =\|\boldsymbol{\tau}(t)\|_{L^{2}(\Omega)}^{2}=\frac{1}{|\Omega|}\left(\int_{\Omega} \mathbf{u}\right)^{2}=\frac{1}{|\Omega|}\left(\int_{0}^{t} \int_{\Omega} \partial_{t} \mathbf{u}(s) \mathrm{d} s+\boldsymbol{\tau}(0)|\Omega|\right)^{2} \\
& \leq 2\left(\left\|\boldsymbol{\tau}_{0}\right\|_{L^{2}(\Omega)}^{2}+t \int_{0}^{t}\left\|\partial_{t} \mathbf{u}(s)\right\|_{L^{2}(\Omega)}^{2} \mathrm{~d} s\right) \\
& \leq 2\left(\left\|\boldsymbol{\tau}_{0}\right\|_{L^{2}(\Omega)}^{2}+T \int_{0}^{t} E(s) \mathrm{d} s\right)
\end{aligned}
$$

Furthermore since $\mathbf{b} \mapsto\left\||\mathbf{b} \||\right.$ is a norm on $\mathcal{E}_{1}$ that is equivalent to the norm $\| \cdot \|_{H^{1}(\Omega)}(c f[5])$, there exists a constant $C$ such that:

$$
\forall \mathbf{b} \in \mathcal{E}_{1}, \quad\|\mathbf{b}\|_{H^{1}(\Omega)}^{2} \leq C\|\mid \mathbf{b}\| \|^{2} .
$$

Then from (28) the following estimates holds:

$$
\begin{aligned}
\|\mathbf{u}(t)\|_{H^{1}(\Omega)}^{2} & \leq 3\left(2\left(\left\|\boldsymbol{\tau}_{0}\right\|_{L^{2}(\Omega)}^{2}+T \int_{0}^{t} E(s) \mathrm{d} s\right)+C+C\|\| \mathbf{d}(t)\|\|^{2}\right) \\
& \leq C\left(1+\int_{0}^{t} E(s) \mathrm{d} s+E(t)\right)
\end{aligned}
$$


where $C$ is a non negative constant depending on the data.

From estimate (27) we then have:

$$
\begin{aligned}
E(t) \leq a_{0} & +\frac{1}{2 \rho_{S}}\|\mathbf{f}\|_{L^{2}\left(0, T ; L^{2}(\Omega)\right)}^{2}+\int_{0}^{t} E(s) \mathrm{d} s+\frac{\gamma}{2}\|\mathbf{g}(t)\|_{H^{-1 / 2}(\partial \Omega)}^{2}+\frac{1}{2 \gamma}\|\mathbf{u}(t)\|_{H^{1}(\Omega)}^{2} \\
& +\frac{C}{2}\left(\left\|\partial_{t} \mathbf{g}(s)\right\|_{L^{2}\left(0, T ; H^{-1 / 2}(\partial \Omega)\right)}^{2}+\int_{0}^{t}\|\mathbf{u}(s)\|_{H^{1}(\Omega)}^{2} \mathrm{~d} s\right)
\end{aligned}
$$

where $\gamma$ is a postive constant. Choosing $\gamma$ large enough and using (29) we obtain

$$
E(t) \leq A_{1}+A_{2} \int_{0}^{t} E(s) \mathrm{d} s+A_{3} \int_{0}^{t} \int_{0}^{s} E(r) \mathrm{d} r \mathrm{~d} s
$$

where $A_{i}$ are constants depending on the data (initial data, forces, $\Omega, T$ ). Remark that $A_{2}>0$. We then apply the Gronwall Lemma to the function $t \mapsto E(t)+\frac{A_{3}}{A_{2}} \int_{0}^{t} E(s) \mathrm{d} s$, assuming that

$$
\mathbf{f} \in L^{2}\left(0, T ; L^{2}(\Omega)\right) \quad \text { and } \quad \mathbf{g} \in H^{1}\left(0, T ; H^{-1 / 2}(\partial \Omega)\right)
$$

This gives bounds on $\partial_{t} \mathbf{u}$ in $L^{\infty}\left(0, T ; L^{2}(\Omega)\right)$ and $\mathbf{d}$ in $L^{\infty}\left(0, T ; H^{1}(\Omega)\right)$. From these bounds and assuming that $\mathbf{d} \in C^{0}\left([0, T] ; \mathbf{Y}_{0}\right)$ we obtain thanks to (8) and (9) applied to $\mathbf{v}=\partial_{t} \mathbf{u}$ that $\dot{\boldsymbol{\tau}}$, $\dot{\theta}, \partial_{t} \mathbf{d}$ are bounded respectively in $L^{\infty}(0, T), L^{\infty}(0, T)$ and $L^{\infty}\left(0, T ; L^{2}(\Omega)\right)$. Consequently any solution $(\boldsymbol{\tau}, \theta, \mathbf{d})$ of $(20)-(23)-(25)$ is bounded, at least formally, in $W^{1, \infty}(0, T) \times W^{1, \infty}(0, T) \times$ $\left(W^{1, \infty}\left(0, T ; L^{2}(\Omega)\right) \cap L^{\infty}\left(0, T ; H^{1}(\Omega)\right)\right)$.

This is in those energy spaces we are going to work.

\section{Main results}

We now state existence and uniqueness results.

Theorem 1 Let $\boldsymbol{\tau}_{0}, \boldsymbol{\tau}_{1}$ be given in $\mathbb{R}^{2}, \theta_{0}, \theta_{1}$ be real numbers, $\mathbf{d}_{0} \in \mathbf{Y}_{1}, \mathbf{d}_{1} \in L^{2}(\Omega)$, $\mathbf{f} \in$ $L_{l o c}^{2}\left(0,+\infty ; L^{2}(\Omega)\right)$ and $\mathbf{g} \in H_{l o c}^{1}\left(0,+\infty ; H^{-1 / 2}(\partial \Omega)\right) \cap L_{l o c}^{2}\left(0,+\infty ; L^{1}(\partial \Omega)\right)$. There exists $T^{*}>0$ such that for all $T<T^{*}$ there exists a unique triplet $(\boldsymbol{\tau}, \theta, \mathbf{d})$ satisfying

$$
\begin{aligned}
& \left\{\begin{array}{l}
\boldsymbol{\tau} \in H^{2}(0, T), \theta \in H^{2}(0, T), \\
\mathbf{d} \in H^{2}\left(0, T ;\left(H^{1}(\Omega)\right)^{\prime}\right) \cap W^{1, \infty}\left(0, T ; L^{2}(\Omega)\right) \cap L^{\infty}\left(0, T ; H^{1}(\Omega)\right) \\
\mathbf{d} \in C^{0}\left([0, T] ; \mathbf{Y}_{0}\right) \\
\text { such that } \\
m \ddot{\boldsymbol{\tau}}=\int_{\Omega} \mathbf{f}+\int_{\partial \Omega} \mathbf{g} \quad \text { in } L^{2}(0, T) \\
\frac{d}{d t}\left(J \dot{\theta} \overrightarrow{e_{z}}+\rho_{S} \int_{\Omega} \mathbf{d} \wedge \partial_{t} \mathbf{d}\right)=\int_{\Omega} R_{\theta}(\overrightarrow{G \xi}+\mathbf{d}) \wedge \mathbf{f}+\int_{\partial \Omega} R_{\theta}(\overrightarrow{G \xi}+\mathbf{d}) \wedge \mathbf{g} \quad \text { in } \mathcal{D}^{\prime}(0, T) \\
\forall \mathbf{b} \in \mathcal{E}_{1}, \rho_{S}\left(\int_{\Omega} \partial_{t t} \mathbf{d} \cdot \mathbf{b}+2 \dot{\theta} \int_{\Omega} \partial_{t} \mathbf{d} \wedge \mathbf{b}+\ddot{\theta} \int_{\Omega} \mathbf{d} \wedge \mathbf{b}-(\dot{\theta})^{2} \int_{\Omega}(\overrightarrow{G \xi}+\mathbf{d}) \cdot \mathbf{b}\right) \\
\quad+\int_{\Omega} \bar{\sigma}(\mathbf{d}): \nabla \mathbf{b}=\int_{\Omega} \mathbf{f} \cdot R_{\theta} \mathbf{b}+\int_{\partial \Omega} \mathbf{g} \cdot R_{\theta} \mathbf{b} \quad \text { in } \mathcal{D}^{\prime}(0, T) \\
\text { together with } \boldsymbol{\tau}(0)=\boldsymbol{\tau}_{0}, \dot{\boldsymbol{\tau}}(0)=\boldsymbol{\tau}_{1}, \theta(0)=\theta_{0}, \dot{\theta}(0)=\theta_{1}, \mathbf{d}(0)=\mathbf{d}_{0} \text { and } \partial_{t} \mathbf{d}(0)=\mathbf{d}_{1}
\end{array}\right. \\
& \text { Moreover the following alternative holds: } \\
& \quad T^{*}=+\infty \text { or } \\
& \lim _{t \longrightarrow T^{*}} \int_{\Omega}(\overrightarrow{G \xi}+\mathbf{d}) \cdot \overrightarrow{G \xi}=0 .
\end{aligned}
$$


This result is equivalent to:

Theorem 2 Let $\mathbf{u}_{0} \in H^{1}(\Omega)$ such that $\mathbf{u}_{0}+\overrightarrow{O \xi} \in \mathbf{X}_{1}$ and $\mathbf{u}_{1} \in L^{2}(\Omega)$. Assume that $\mathbf{f} \in$ $L_{l o c}^{2}\left(0,+\infty ; L^{2}(\Omega)\right)$ and $\mathbf{g} \in H_{l o c}^{1}\left(0,+\infty ; H^{-1 / 2}(\partial \Omega)\right) \cap L_{l o c}^{2}\left(0,+\infty ; L^{1}(\partial \Omega)\right)$. There exists $T^{*}>0$ such that for all $T<T^{*}$ there exists a unique

$$
\mathbf{u} \in H^{2}\left(0, T ;\left(H^{1}(\Omega)\right)^{\prime}\right) \cap W^{1, \infty}\left(0, T ; L^{2}(\Omega)\right) \cap L^{\infty}\left(0, T ; H^{1}(\Omega)\right)
$$

and

solution of

$$
\phi=\mathbf{u}+\operatorname{Id}_{\mathbb{R}^{2}} \in C^{0}\left([0, T] ; \mathbf{X}_{0}\right)
$$

$$
\left\{\begin{array}{l}
\forall \mathbf{v} \in H^{1}(\Omega), \quad \rho_{S} \frac{d}{d t}\left(\int_{\Omega} \partial_{t} \mathbf{u} \cdot \mathbf{v}\right)+\int_{\Omega} \bar{\sigma}(\mathbf{d}): \nabla \mathbf{b}=\int_{\Omega} \mathbf{f} \cdot \mathbf{v}+\int_{\partial \Omega} \mathbf{g} \cdot \mathbf{v} \quad \text { in } \quad \mathcal{D}^{\prime}(0, T), \\
\text { with } \mathbf{b} \text { associated to } \mathbf{v} \text { though }(9), \mathbf{d} \text { associated to } \mathbf{u} \text { and defined by Proposition } 2, \\
\mathbf{u}(\cdot, t=0)=\mathbf{u}_{0}, \quad \partial_{t} \mathbf{u}(\cdot, t=0)=\mathbf{u}_{1} .
\end{array}\right.
$$

Moreover the following alternative holds

$$
\begin{aligned}
& -T^{*}=+\infty \text { or } \\
& -\lim _{t \longrightarrow T^{*}} \int_{\Omega}(\overrightarrow{G \xi}+\mathbf{d}) \cdot \overrightarrow{G \xi}=0 .
\end{aligned}
$$

The proof of these theorems will be split into several steps. First we prove that there exists a local solution to (30), then that this solution is unique. The proof of the existence of at least one solution of (30) is based on a Galerkin method. We then extend this solution until $\int_{\Omega}(\overrightarrow{G \xi}+\mathbf{d}) \cdot \overrightarrow{G \xi}>0$ remains valid. To conclude we prove that Theorem 1 is equivalent to Theorem 2.

\section{Proof of Theorem 1}

\subsection{Existence of a local solution}

The first remark is that the existence and uniqueness of $\tau$ is straightforward. Thus we will look at the coupled system describing the dynamics of $\theta$ and $\mathbf{d}$.

\subsubsection{Modal approximation}

In many applications, it is sufficient to consider that $\mathbf{d}$ belongs to a well chosen space of finite dimension, and thus to approximate our system by a discrete one. A natural basis is, in the case we deal with, the family of eigenfunctions associated with all non negative eigenvalues of the linearized elasticity operator $\bar{\Sigma}$. This family of eigenfunctions is a basis of $\mathcal{E}_{1}$. Indeed let us consider the operator of linearized elasticity $\bar{\Sigma}$ with homogeneous Neumann boundary conditions. This is a selfadjoint operator of $L^{2}(\Omega)$ whose inverse is a bounded operator from $\mathcal{E}_{0}$ onto $\mathcal{E}_{0}$. We define the eigenfunctions $\psi_{i}, i \in \mathbb{N}$ by:



The family $\left(\psi_{i}\right)_{i \in \mathbb{N}}$ is a basis of $H^{1}(\Omega)$ that we choose othonormal in $L^{2}(\Omega)$. We denote by $\psi_{0,1}=\frac{1}{|\Omega|^{1 / 2}} \frac{\overrightarrow{e_{x}}}{\left\|\overrightarrow{e_{x}}\right\|}, \psi_{0,2}=\frac{1}{|\Omega|^{1 / 2}} \frac{\overrightarrow{e_{y}}}{\left\|\overrightarrow{e_{y}}\right\|}$, and $\psi_{0,3}=\overrightarrow{e_{z}} \wedge \overrightarrow{G \xi} /\|\overrightarrow{G \xi}\|_{L^{2}(\Omega)}$ the eigenvectors associated with the eigenvalue 0 with multiplicity 3 . These eigenfunctions are translations and infinitesimal 
rotations. This family of eigenfunctions is a basis of $\operatorname{Ker}(\bar{\Sigma})$. Consequently $\mathcal{E}_{1}=<\psi_{i}>_{i \in \mathbb{N}^{*}}$, and we approximate $\mathbf{d}$ by a linear combinaison of a finite number $N \in \mathbb{N}^{*}$ of modal functions: $\mathbf{d}_{N}=\sum_{j=1}^{N} \alpha_{j}(t) \psi_{j}$.

This will be used in what follows to prove the existence of a solution of our problem: the family of eigenfunctions $\left(\psi_{i}\right)_{i \in \mathbb{N}^{*}}$ will be chosen as a Galerkin basis of $\mathcal{E}_{1}$.

In this subsection we prove that the approximate problem is wellposed and that it converges towards the continuous one. We set $\mathbf{d}_{N}(\xi, t)=\sum_{j=1}^{N} \alpha_{j}(t) \psi_{j}(\xi)$. The problem (23)-(25) is approxiamated by the following discrete problem: find $\left(\theta_{N}, \mathbf{d}_{N}\right)$ satisfying

$$
\left\{\begin{array}{r}
\frac{d}{d t}\left(J_{N}(t) \dot{\theta}_{N}\right)+\rho_{S} \int_{\Omega} \mathbf{d}_{N} \wedge \partial_{t t} \mathbf{d}_{N}=\int_{\Omega} R_{\theta_{N}}\left(\overrightarrow{G \xi}+\mathbf{d}_{N}\right) \wedge \mathbf{f}+\int_{\partial \Omega} R_{\theta_{N}}\left(\overrightarrow{G \xi}+\mathbf{d}_{N}\right) \wedge \mathbf{g} \\
1 \leq i \leq N, \quad \rho_{S}\left(\int_{\Omega} \partial_{t t} \mathbf{d}_{N} \cdot \psi_{i}+2 \dot{\theta}_{N} \int_{\Omega} \partial_{t} \mathbf{d}_{N} \wedge \psi_{i}+\ddot{\theta}_{N} \int_{\Omega} \mathbf{d}_{N} \wedge \psi_{i}-\left(\dot{\theta}_{N}\right)^{2} \int_{\Omega}\left(\overrightarrow{G \xi}+\mathbf{d}_{N}\right) \cdot \psi_{i}\right) \\
\quad+\int_{\Omega} \bar{\sigma}\left(\mathbf{d}_{N}\right): \nabla \psi_{i}=\int_{\Omega} \mathbf{f} \cdot R_{\theta_{N}} \psi_{i}+\int_{\partial \Omega} \mathbf{g} \cdot R_{\theta_{N}} \psi_{i}
\end{array}\right.
$$

together with initial conditions:

$$
\left\{\begin{array}{cl}
\theta_{N}(0)=\theta_{0}, & \mathbf{d}_{N}(\cdot, t=0)=\mathbf{d}_{N 0}, \\
\dot{\theta}_{N}(0)=\theta_{1}, & \partial_{t} \mathbf{d}_{N}(\cdot, t=0)=\mathbf{d}_{N 1},
\end{array}\right.
$$

where $\mathbf{d}_{N 0}=\Pi_{N}^{0}\left(\mathbf{d}_{0}\right)$ (resp. $\mathbf{d}_{N 1}=\Pi_{N}^{0}\left(\mathbf{d}_{1}\right)$ ) with $\Pi_{N}^{0}$ denoting the $L^{2}(\Omega)$-orthogonal projector operator on $\mathcal{E}^{N}=<\psi_{i} ; 1 \leq i \leq N>$. Since $\left(\psi_{i}\right)_{i \in \mathbb{N}}$ is an hilbertian basis of $\left(\mathcal{E}_{1},((.,)).\right)$ and of $\left(L^{2}(\Omega), \int_{\Omega} \cdot.\right)$, we have as $N$ goes to infinity:

$$
\mathbf{d}_{N 0} \longrightarrow \mathbf{d}_{0} \text { in } H^{1}(\Omega) \text { and } \quad \mathbf{d}_{N 1} \longrightarrow \mathbf{d}_{1} \text { in } L^{2}(\Omega) .
$$

The discrete system can be written as

$$
A(X) \ddot{X}=h(t, X, \dot{X})
$$

where $X^{T}=\left[\theta, \alpha_{1}, \ldots, \alpha_{N}\right] \in \mathbb{R}^{N+1}$, and where, using the fact that $\left(\psi_{i}\right)_{i}$ is orthonormal in $L^{2}(\Omega)$, the mass matrix $A(X) \in \mathcal{M}_{N+1}(\mathbb{R})$ is given by:

$$
A(X)=\left(\begin{array}{cc}
J_{N} & \left(\rho_{S} \int_{\Omega} \mathbf{d}_{N} \wedge \psi_{i}\right)_{1 \leq i \leq N}^{T} \\
\left(\rho_{S} \int_{\Omega} \mathbf{d}_{N} \wedge \psi_{i}\right)_{1 \leq i \leq N} & \left(\rho_{S} \delta_{i, j}\right)_{1 \leq i, j \leq N}
\end{array}\right) .
$$

At this step of the proof the fact that the Galerkin basis is a familly of eigenvectors associated to the linear elasticity operator plays no role. It will in the next subsection. We prove now that the mass matrix is invertible if $\int_{\Omega}\left(\overrightarrow{G \xi}+\mathbf{d}_{N}\right) \cdot \overrightarrow{G \xi} \neq 0$. Indeed, for $B^{T}=\left(\beta, \beta_{1}, \ldots, \beta_{N}\right) \in \mathbb{R}^{N+1}$, and setting $\mathbf{b}=\sum_{i=1}^{N} \beta_{i} \psi_{i}$, we have:

$$
\begin{aligned}
B^{T} & \cdot A(X) B=J_{N} \beta^{2}+2 \rho_{S} \beta \int_{\Omega} \mathbf{d}_{N} \wedge \mathbf{b}+\rho_{S} \int_{\Omega}|\mathbf{b}|^{2} \\
& =\rho_{S} \int_{\Omega}\left[\beta \overrightarrow{e_{z}} \wedge R_{\theta_{N}}\left(\overrightarrow{G \xi}+\mathbf{d}_{N}\right)\right]^{2}+2 \rho_{S} \int_{\Omega}\left[\beta \overrightarrow{e_{z}} \wedge R_{\theta_{N}}\left(\overrightarrow{G \xi}+\mathbf{d}_{N}\right)\right] \cdot R_{\theta_{N}} \mathbf{b}+\rho_{S} \int_{\Omega}\left|R_{\theta_{N}} \mathbf{b}\right|^{2} \\
& =\rho_{S}\left\|\beta \overrightarrow{e_{z}} \wedge R_{\theta_{N}}\left(\overrightarrow{G \xi}+\mathbf{d}_{N}\right)+R_{\theta_{N}} \mathbf{b}\right\|_{L^{2}(\Omega)}^{2} \geq 0
\end{aligned}
$$


Thus remembering that (12) holds if $\int_{\Omega}\left(\overrightarrow{G \xi}+\mathbf{d}_{N}\right) \cdot \overrightarrow{G \xi} \neq 0$ we deduce that $A(X)$ is a positive definite matrix.

We now apply the Carathéodory Theorem (cf [3] page 43) in $\mathcal{U} \times \mathbb{R}^{N+1}$ with:

$$
\mathcal{U}=\left\{\left(\theta, \alpha_{1}, \ldots, \alpha_{N}\right) \in \mathbb{R} / 2 \pi \mathbb{Z} \times \mathbb{R}^{N} ; \sum_{i=1}^{N} \alpha_{i} \psi_{i} \in \mathbf{Y}_{0}\right\}
$$

Then the system of $\operatorname{ODE}(35)$ has a unique solution $X=\left(\theta, \alpha_{1}, \ldots \alpha_{N}\right) \in\left[H^{2}\left(0, T_{N}\right)\right]^{N+1}$, $T_{N}>0$. In particular, $\mathbf{d}_{N}=\sum_{i=1}^{N} \alpha_{i} \psi_{i} \in C^{0}\left(\left[0, T_{N}\right] ; \mathbf{Y}_{0}\right)$. Moreover the solution satisfies energy estimates. Indeed multiplying (23) by $\theta_{N}$ then (25) by $\alpha_{i}$ for $1 \leq i \leq N$ and summing up these contributions, we obtain, as for the continuous problem, that $\mathbf{v}_{N}=\dot{\theta}_{N} \overrightarrow{e_{z}} \wedge R_{\theta_{N}}\left(\overrightarrow{G \xi}+\mathbf{d}_{N}\right)+$ $R_{\theta_{N}} \partial_{t} \mathbf{d}_{N}$ is bounded in $L^{\infty}\left(0, T_{N} ; L^{2}(\Omega)\right)$ independently of $N$ and that:

$$
\left\|\mathbf{d}_{N}\right\|_{L^{\infty}\left(0, T_{N} ; H^{1}(\Omega)\right)} \leq C
$$

with $C$ depending only on the data and independent of $N$. Let $\delta$ be a real number such that $\int_{\Omega}\left(\overrightarrow{G \xi}+\mathbf{d}_{0}\right) \cdot \overrightarrow{G \xi}>\delta>0$. For $N$ sufficiently large we have $\int_{\Omega}\left(\overrightarrow{G \xi}+\mathbf{d}_{N_{0}}\right) \cdot \overrightarrow{G \xi}>\delta$. If we set

$$
\mathbf{Y}_{0}^{\delta}=\left\{\mathbf{b} \in L^{2}(\Omega), \int_{\Omega}(\overrightarrow{G \xi}+\mathbf{b}) \cdot \overrightarrow{G \xi} \geq \delta\right\}
$$

we deduce from the continuity in time of $\mathbf{d}_{N}$ that there exists $T_{N}^{\delta} \leq T_{N}$ such that $\mathbf{d}_{N} \in$ $C^{0}\left(\left[0, T_{N}^{\delta}\right] ; \mathbf{Y}_{0}^{\delta}\right)$. Then expressing $\dot{\theta}_{N}$ and $\partial_{t} \mathbf{d}_{N}$ with respect to $\mathbf{v}_{N}$ (see (8) and (9)) we have that $\dot{\theta}_{N}$ and $\partial_{t} \mathbf{d}_{N}$ are bounded respectively in $L^{\infty}\left(0, T_{N}^{\delta}\right)$ and $L^{\infty}\left(0, T_{N}^{\delta} ; L^{2}(\Omega)\right)$, and these bounds are independent of $N$ but depend on $\delta$. Consequently, there exists a time $T^{\delta}$ such that $\mathbf{d}_{N}$ is bounded in $C^{0}\left(\left[0, T^{\delta}\right] ; \mathbf{Y}_{0}^{\delta}\right)$ independently of $N$. Thus $T_{N}^{\delta}$ can be chosen independently of $N$.

Remark 4 Using (8) and (9), we obtain by (36) that for all $t \in\left[0, T^{\delta}\right]$ :

$$
B^{T} \cdot A(X) B=\rho_{S}\left\|\beta \overrightarrow{e_{z}} \wedge R_{\theta_{N}}\left(\overrightarrow{G \xi}+\mathbf{d}_{N}\right)+R_{\theta_{N}} \mathbf{b}\right\|_{L^{2}(\Omega)}^{2} \geq \alpha(\delta)|B|_{\mathbb{R}^{N+1}}^{2}
$$

where $|\cdot|_{\mathbb{R}^{N+1}}$ is the euclidian norm in $\mathbb{R}^{N+1}$ and where $\alpha(\delta)$ is of order $\delta$.

In all what follows we fix $\delta>0$ satisfying $\int_{\Omega}\left(\overrightarrow{G \xi}+\mathbf{d}_{0}\right) \cdot \overrightarrow{G \xi}>\delta$ and we work on $\left[0, T^{\delta}\right]$ such that $\mathbf{d}_{N}$ is bounded in $C^{0}\left(\left[0, T^{\delta}\right] ; \mathbf{Y}_{0}^{\delta}\right)$ independently of $N$. For simplicity we set $T=T^{\delta}$.

\subsubsection{Additional estimate}

The previous estimates enable us to pass to the limit in a weak sense in the discrete system. Nevertheless, we are not able to identify the limit function and nor prove that it corresponds to the solution of the continuous problem. For instance we know that $\dot{\theta}_{N}$ and $\left(\dot{\theta}_{N}\right)^{2}$ converge in $L^{\infty}(0, T)$ weak-star respectively to $\dot{\theta}$ and $\chi$ but we do not know whether $\chi=(\dot{\theta})^{2}$. We are thus going to derive additional estimates on $\ddot{\theta}_{N}$ and $\partial_{t t} \mathbf{d}_{N}$ that will provide us compactness properties. We prove that $\ddot{\theta}_{N}$ is bounded in $L^{\infty}(0, T)$ and that $\partial_{t t} \mathbf{d}_{N}$ is bounded in $L^{2}\left(0, T ;\left(H^{1}(\Omega)\right)^{\prime}\right)$. The analysis uses a duality argument and relies on the "special" Galerkin basis we have chosen ([10]).

First we recall the properties of $\Pi_{N}^{0}$ the $L^{2}$-projector on $\mathcal{E}_{N}$ and of $\Pi_{N}^{1}$ the projector on $\mathcal{E}_{N}$ with respect to the semi-norm $\||\cdot \||$. Thanks to the choice of the special basis, we have that

$$
\forall \mathbf{b} \in H^{1}(\Omega), \quad \Pi_{N}^{0}(\mathbf{b})=\Pi_{N}^{1}(\mathbf{b}) .
$$


Consequently, the restriction of $\Pi_{N}^{0}$ to $H^{1}(\Omega)$ is stable with respect to the semi-norm $\||\cdot|\|$. Moreover since \|\|$\cdot \|$ is a norm on $\mathcal{E}_{1}$ equivalent to the $H^{1}$-norm, there exists constants such that:

$$
\forall \mathbf{b} \in H^{1}(\Omega), \quad C\left\|\Pi_{N}^{0}(\mathbf{b})\right\|_{H^{1}(\Omega)} \leq\|\| \Pi_{N}^{0}(\mathbf{b})\|\mid \leq\| \mathbf{b}\left\|\leq C^{\prime}\right\| \mathbf{b} \|_{H^{1}(\Omega)} .
$$

This implies that the restriction of $\Pi_{N}^{0}$ to $H^{1}(\Omega)$ is stable with respect to the $H^{1}$-norm.

At this stage an important remark is that if the estimates are carried out without paying attention, we obtain the desired result under a condition of smallness of the data (as annonced in $[9]$ ). We only explain quickly why (since the same kind of estimates will be detailed right after). Indeed for any $\mathbf{b}$ in $H^{1}(\Omega)$ and with $\mathbf{b}_{N}=\Pi_{N}^{0}(\mathbf{b})$ we have from the dynamic of $\mathbf{d}_{N}$ (see (33)):

$$
\begin{aligned}
& \rho_{S} \int_{\Omega} \partial_{t t} \mathbf{d}_{N} \cdot \mathbf{b}=\rho_{S} \int_{\Omega} \partial_{t t} \mathbf{d}_{N} \cdot \mathbf{b}_{N}= \\
&-\rho_{S}\left(2 \dot{\theta}_{N} \int_{\Omega} \partial_{t} \mathbf{d}_{N} \wedge \mathbf{b}_{N}+\ddot{\theta}_{N} \int_{\Omega} \mathbf{d}_{N} \wedge \mathbf{b}_{N}-\left(\dot{\theta}_{N}\right)^{2} \int_{\Omega}\left(\overrightarrow{G \xi}+\mathbf{d}_{N}\right) \cdot \mathbf{b}_{N}\right) \\
& \quad-\left(\left(\mathbf{d}_{N}, \mathbf{b}_{N}\right)\right)+\int_{\Omega} \mathbf{f} \cdot R_{\theta_{N}} \mathbf{b}_{N}+\int_{\partial \Omega} \mathbf{g} \cdot R_{\theta_{N}} \mathbf{b}_{N} .
\end{aligned}
$$

Using the energy estimates and the stability of the orthogonal projector $\Pi_{N}^{0}$ in the $L^{2}$-norm, as well as in the $H^{1}$-norm, we obtain by duality

$$
\left\|\partial_{t t} \mathbf{d}_{N}\right\|_{L^{2}\left(0, T ;\left(H^{1}(\Omega)\right)^{\prime}\right)} \leq C_{1}+C_{2}\left\|\ddot{\theta}_{N}\right\|_{L^{2}(0, T)},
$$

where $C_{i}, i=1,2$ depend on the data. The equation describing the dymanic of $\theta_{N}$ also couple $\ddot{\theta}_{N}$ and $\partial_{t t} \mathbf{d}_{N}$ :

$$
\begin{gathered}
J_{N}(t) \ddot{\theta}_{N}=\rho_{S} \int_{\Omega} \partial_{t t} \mathbf{d}_{N} \wedge \mathbf{d}_{N}+\int_{\Omega} R_{\theta_{N}}\left(\overrightarrow{G \xi}+\mathbf{d}_{N}\right) \wedge \mathbf{f}+\int_{\partial \Omega} R_{\theta_{N}}\left(\overrightarrow{G \xi}+\mathbf{d}_{N}\right) \wedge \mathbf{g} \\
-2 \rho_{S} \dot{\theta}_{N} \int_{\Omega}\left(\overrightarrow{G \xi}+\mathbf{d}_{N}\right) \cdot \partial_{t} \mathbf{d}_{N} .
\end{gathered}
$$

For any $t \in[0, T], \mathbf{d}_{N}(t) \in \mathbf{Y}_{0}^{\delta}$, and thus:

$$
0<\sqrt{\rho_{S}} \delta \leq \sqrt{\rho_{S}} \int_{\Omega}\left(\overrightarrow{G \xi}+\mathbf{d}_{N}\right) \cdot \overrightarrow{G \xi} \leq \sqrt{\rho_{S}}\left\|\overrightarrow{G \xi}+\mathbf{d}_{N}\right\|_{L^{2}(\Omega)}\|\overrightarrow{G \xi}\|_{L^{2}(\Omega)} \leq \sqrt{J_{N}(t)}\|\overrightarrow{G \xi}\|_{L^{2}(\Omega)}
$$

Using the energy estimates, this leads to

$$
\left\|\ddot{\theta}_{N}\right\|_{L^{2}(0, T)} \leq C_{3}+C_{4}\left\|\partial_{t t} \mathbf{d}_{N}\right\|_{L^{2}\left(0, T ;\left(H^{1}(\Omega)\right)^{\prime}\right)}
$$

where $C_{i}, i=3,4$ depend on the data, on $\delta$ but are independent of $N$. The inequalities (40) and (41), finally give

$$
\left\|\partial_{t t} \mathbf{d}_{N}\right\|_{L^{2}\left(0, T ;\left(H^{1}(\Omega)\right)^{\prime}\right)} \leq C_{5}+C_{6}\left\|\partial_{t t} \mathbf{d}_{N}\right\|_{L^{2}\left(0, T ;\left(H^{1}(\Omega)\right)^{\prime}\right)} .
$$

with $C_{i}, i=5,6$ depending on the data, on $\delta$ but are independent of $N$. Thus if the data are small enough, $C_{6}$ can be chosen strictly less than 1 and we obtain the desired estimate. Since we do not want these kinds of restrictions we are going to estimate separatly the high frequencies and the low frequencies of $\partial_{t t} \mathbf{d}_{N}$. Let us consider $N_{0} \in \mathbb{N}^{*}$ that will be chosen later on and such that $N \geq N_{0}$. We separate $\mathbf{d}_{N}$ into two parts:

$$
\mathbf{d}_{N}=\beta_{N}+\omega_{N} \quad \text { where } \quad \beta_{N}=\sum_{i=1}^{N_{0}-1} \alpha_{i} \psi_{i} \quad \text { and } \quad \omega_{N}=\sum_{i=N_{0}}^{N} \alpha_{i} \psi_{i}
$$

We set $H f^{N_{0}}=\left\langle\psi_{i} ; i \in \mathbb{N}, i \geq N_{0}\right\rangle$. 


\section{Estimate on the high frequencies}

We introduce the orthogonal projection $\Pi_{N}^{0, h f}$ from $L^{2}(\Omega)$ on $\mathcal{E}^{N} \cap H f^{N_{0}}$, for which we have

$$
\forall \mathbf{b} \in L^{2}(\Omega), \quad \int_{\Omega} \partial_{t t} \mathbf{d}_{N} \cdot \Pi_{N}^{0, h f}(\mathbf{b})=\int_{\Omega} \partial_{t t} \Pi_{N}^{0, h f}\left(\mathbf{d}_{N}\right) \cdot \mathbf{b}=\int_{\Omega} \partial_{t t} \omega_{N} \cdot \mathbf{b} .
$$

Taking $\mathbf{b}_{N}=\Pi_{N}^{0, h f}(\mathbf{b})$ as a test function in (39) with $\mathbf{b} \in H^{1}(\Omega)$ and using (42) the following equality holds:

$$
\begin{array}{r}
\rho_{S} \int_{\Omega} \partial_{t t} \omega_{N} \cdot \mathbf{b}=-\rho_{S}\left(\ddot{\theta}_{N} \int_{\Omega} \mathbf{d}_{N} \wedge \mathbf{b}_{N}+2 \dot{\theta}_{N} \int_{\Omega} \partial_{t} \mathbf{d}_{N} \wedge \mathbf{b}_{N}-\left(\dot{\theta}_{N}\right)^{2} \int_{\Omega}\left(\overrightarrow{G \xi}+\mathbf{d}_{N}\right) \cdot \mathbf{b}_{N}\right) \\
-\left(\left(\mathbf{d}_{N}, \mathbf{b}_{N}\right)\right)+\int_{\Omega} \mathbf{f} \cdot R_{\theta_{N}} \mathbf{b}_{N}+\int_{\partial \Omega} \mathbf{g} \cdot R_{\theta_{N}} \mathbf{b}_{N} .
\end{array}
$$

Thus:

$$
\begin{aligned}
& \left|\int_{\Omega} \partial_{t t} \omega_{N} \cdot \mathbf{b}\right| \leq\left[\left|\ddot{\theta}_{N}\right|\left\|\mathbf{d}_{N}\right\|_{L^{2}(\Omega)}+2\left|\dot{\theta}_{N}\right|\left\|\partial_{t} \mathbf{d}_{N}\right\|_{L^{2}(\Omega)}+\left|\dot{\theta}_{N}\right|^{2}\left\|\overrightarrow{G \xi}+\mathbf{d}_{N}\right\|_{L^{2}(\Omega)}\right. \\
& \left.\quad+\frac{1}{\rho_{S}}\|\mathbf{f}\|_{L^{2}(\Omega)}\right]\left\|\mathbf{b}_{N}\right\|_{L^{2}(\Omega)}+\frac{C}{\rho_{S}}\|\mathbf{g}\|_{H^{-1 / 2}(\partial \Omega)}\left\|\mathbf{b}_{N}\right\|_{H^{1}(\Omega)}+\frac{1}{\rho_{S}}\left\|\mathbf{d}_{N}\right\|\left|\left\||| \mathbf{b}_{N}\right\| \| .\right.
\end{aligned}
$$

Thanks to the choice of the special basis and to the equivalence of the norm $|\|\cdot\|| \|$ and $\|\cdot\|_{H^{1}(\Omega)}$ on $\mathcal{E}_{1}$ we have that the restriction of $\Pi_{N}^{0, h f}$ to $H^{1}(\Omega)$ is stable with respect to the $H^{1}$-norm. Moreover,

$$
\forall \mathbf{b} \in H^{1}(\Omega), \quad\left\|\Pi_{N}^{0, h f}(\mathbf{b})\right\|_{L^{2}(\Omega)} \leq \frac{1}{\sqrt{\lambda_{N_{0}}}}\left\|\Pi_{N}^{0, h f}(\mathbf{b})\right\| \leq \frac{1}{\sqrt{\lambda_{N_{0}}}}\|\mathbf{b}\| \mid \leq \frac{C}{\sqrt{\lambda_{N_{0}}}}\|\mathbf{b}\|_{H^{1}(\Omega)}
$$

This leads to:

$$
\begin{aligned}
&\left|\int_{\Omega} \partial_{t t} \omega_{N} \cdot \mathbf{b}\right| \leq\left(2\left|\dot{\theta}_{N}\right|\left\|\partial_{t} \mathbf{d}_{N}\right\|_{L^{2}(\Omega)}+\left|\ddot{\theta}_{N}\right|\left\|\mathbf{d}_{N}\right\|_{L^{2}(\Omega)}+\left|\dot{\theta}_{N}\right|^{2}\left\|\overrightarrow{G \xi}+\mathbf{d}_{N}\right\|_{L^{2}(\Omega)}\right. \\
&\left.+\frac{1}{\rho_{S}}\|\mathbf{f}\|_{L^{2}(\Omega)}\right) \frac{C}{\sqrt{\lambda_{N_{0}}}}\|\mathbf{b}\|_{H^{1}(\Omega)}+C\left(\|\mathbf{g}\|_{H^{-1 / 2}(\partial \Omega)}+\left\|\mathbf{d}_{N}\right\|_{H^{1}(\Omega)}\right)\|\mathbf{b}\|_{H^{1}(\Omega)} .
\end{aligned}
$$

Taking the $L^{2}$-norm in time we get by duality:

$$
\begin{aligned}
& \left\|\partial_{t t} \omega_{N}\right\|_{L^{2}\left(0, T ;\left(H^{1}(\Omega)\right)^{\prime}\right)} \leq \frac{C}{\sqrt{\lambda_{N_{0}}}}\left(2\left\|\dot{\theta}_{N}\right\|_{L^{\infty}(0, T)}\left\|\partial_{t} \mathbf{d}_{N}\right\|_{L^{2}\left(0, T ; L^{2}(\Omega)\right)}\right. \\
& +\left\|\ddot{\theta}_{N}\right\|_{L^{2}(0, T)}\left\|\mathbf{d}_{N}\right\|_{L^{\infty}\left(0, T ; L^{2}(\Omega)\right)}+\left\|\dot{\theta}_{N}\right\|_{L^{\infty}(0, T)}^{2}\left\|\overrightarrow{G \xi}+\mathbf{d}_{N}\right\|_{L^{2}\left(0, T ; L^{2}(\Omega)\right)} \\
& \left.\quad+\frac{1}{\rho_{S}}\|\mathbf{f}\|_{L^{2}\left(0, T ; L^{2}(\Omega)\right)}\right)+C\|\mathbf{g}\|_{L^{2}\left(0, T ; H^{-1 / 2}(\partial \Omega)\right)}+C\left\|\mathbf{d}_{N}\right\|_{L^{2}\left(0, T ; H^{1}(\Omega)\right)} .
\end{aligned}
$$

Now thanks to the energy estimates $\theta_{N}$ is bounded in $W^{1, \infty}(0, T), \mathbf{d}_{N}$ is bounded in $L^{\infty}\left(0, T ; H^{1}(\Omega)\right) \cap$ $W^{1, \infty}\left(0, T ; L^{2}(\Omega)\right)$, those bounds beeing independent of $N$. That yield:

$$
\left\|\partial_{t t} \omega_{N}\right\|_{L^{2}\left(0, T ;\left(H^{1}(\Omega)\right)^{\prime}\right)} \leq C+\frac{C}{\sqrt{\lambda_{N_{0}}}}\left\|\ddot{\theta}_{N}\right\|_{L^{2}(0, T)}
$$

where $C$ depends on the initial data, on the exterior forces and on $\delta$. We remark that unlike inequality (40), in (44) we have a control on the constant in front of $\left\|\ddot{\theta}_{N}\right\|_{L^{2}(0, T)}$ which can be as small as desired by choising $N_{0}$ large enough. 


\section{Estimate on the low frequencies}

We now look at the low frequency part $\beta_{N}$ of $\mathbf{d}_{N}$. We multiply the first equation of (33) by $\ddot{\theta}_{N}$, and choose $\partial_{t t} \beta_{N}$ as a test function in the weak formulation describing the dynamic of the elastic displacement. Summing up those contributions it comes:

$$
\begin{aligned}
J_{N}\left(\ddot{\theta}_{N}\right)^{2}+ & 2 \rho_{S} \ddot{\theta}_{N} \int_{\Omega} \mathbf{d}_{N} \wedge \partial_{t t} \beta_{N}+\rho_{S} \int_{\Omega}\left|\partial_{t t} \beta_{N}\right|^{2} \\
= & \ddot{\theta}_{N}\left(\int_{\Omega} R_{\theta_{N}}\left(\overrightarrow{G \xi}+\mathbf{d}_{N}\right) \wedge \mathbf{f}+\int_{\partial \Omega} R_{\theta_{N}}\left(\overrightarrow{G \xi}+\mathbf{d}_{N}\right) \wedge \mathbf{g}-\rho_{S} \int_{\Omega} \mathbf{d}_{N} \wedge \partial_{t t} \omega_{N}\right. \\
& \left.-2 \rho_{S} \dot{\theta}_{N} \int_{\Omega}\left(\overrightarrow{G \xi}+\mathbf{d}_{N}\right) \cdot \partial_{t} \mathbf{d}_{N}\right)+\left(\int_{\Omega} \mathbf{f} \cdot R_{\theta_{N}} \partial_{t t} \beta_{N}+\int_{\partial \Omega} \mathbf{g} \cdot R_{\theta_{N}} \partial_{t t} \beta_{N}\right. \\
- & \left.\int_{\Omega} \bar{\sigma}\left(\mathbf{d}_{N}\right): \nabla \partial_{t t} \beta_{N}+\rho_{S}\left(\dot{\theta}_{N}\right)^{2} \int_{\Omega}\left(\overrightarrow{G \xi}+\mathbf{d}_{N}\right) \cdot \partial_{t t} \beta_{N}-2 \rho_{S} \dot{\theta}_{N} \int_{\Omega} \partial_{t} \mathbf{d}_{N} \wedge \partial_{t t} \beta_{N}\right)
\end{aligned}
$$

The left hand side of (45) can be written using (36):

$J_{N}\left(\ddot{\theta}_{N}\right)^{2}+2 \rho_{S} \ddot{\theta}_{N} \int_{\Omega} \mathbf{d}_{N} \wedge \partial_{t t} \beta_{N}+\rho_{S} \int_{\Omega}\left|\partial_{t t} \beta_{N}\right|^{2}=\rho_{S}\left\|\ddot{\theta}_{N} \overrightarrow{e_{z}} \wedge R_{\theta_{N}}\left(\overrightarrow{G \xi}+\mathbf{d}_{N}\right)+R_{\theta_{N}} \partial_{t t} \beta_{N}\right\|_{L^{2}(\Omega)}^{2}$.

Let us introduce $\mathbf{v}=\bar{\theta} \overrightarrow{e_{z}} \wedge R_{\theta_{N}}\left(\overrightarrow{G \xi}+\mathbf{d}_{N}\right)+R_{\theta_{N}} \overline{\mathbf{d}} \in L^{2}(\Omega)$, and remember that $\mathbf{d}_{N} \in C^{0}\left([0, T] ; \mathbf{Y}_{0}^{\delta}\right)$, we have thanks to (8) and (9):

$$
\begin{gathered}
|\bar{\theta}| \leq \frac{1}{\delta}\|\overrightarrow{G \xi}\|_{L^{2}(\Omega)}\|\mathbf{v}\|_{L^{2}(\Omega)}, \\
\|\overline{\mathbf{d}}\|_{L^{2}(\Omega)} \leq\left\|\mathbf{v}-\bar{\theta} \overrightarrow{e_{z}} \wedge R_{\theta_{N}}\left(\overrightarrow{G \xi}+\mathbf{d}_{N}\right)\right\|_{L^{2}(\Omega)} \leq\left(1+\frac{C}{\delta}\right)\|\mathbf{v}\|_{L^{2}(\Omega)},
\end{gathered}
$$

because $\mathbf{d}_{N}$ is bounded in $L^{\infty}\left(0, T ; L^{2}(\Omega)\right)$. Thus:

$$
|\bar{\theta}|^{2}+\|\overline{\mathbf{d}}\|_{L^{2}(\Omega)}^{2} \leq C\|\mathbf{v}\|_{L^{2}(\Omega)}^{2},
$$

where $\mathrm{C}$ depends on the data and on $\delta^{-1}$. Thus for $\bar{\theta}=\ddot{\theta}_{N}$ and $\overline{\mathbf{d}}=\partial_{t t} \beta_{N}$ we have

$$
\left|\ddot{\theta}_{N}\right|^{2}+\left\|\partial_{t t} \beta_{N}\right\|_{L^{2}(\Omega)}^{2} \leq C\left\|\ddot{\theta}_{N} \overrightarrow{e_{z}} \wedge R_{\theta_{N}}\left(\overrightarrow{G \xi}+\mathbf{d}_{N}\right)+R_{\theta_{N}} \partial_{t t} \beta_{N}\right\|_{L^{2}(\Omega)}^{2} .
$$

We now estimate the right hand side of (45) (taking into account (46) and (47) to obtain a lower bound for the left hand side). Consequently:

$$
\begin{aligned}
\left|\ddot{\theta}_{N}\right|^{2} & +\left\|\partial_{t t} \beta_{N}\right\|_{L^{2}(\Omega)}^{2} \leq C\left|\ddot{\theta}_{N}\right|\left(\rho_{S}\left\|\mathbf{d}_{N}\right\|_{H^{1}(\Omega)}\left\|\partial_{t t} \omega_{N}\right\|_{\left(H^{1}(\Omega)\right)^{\prime}}\right. \\
& \left.+\left\|\overrightarrow{G \xi}+\mathbf{d}_{N}\right\|_{H^{1}(\Omega)}\left[\|\mathbf{f}\|_{L^{2}(\Omega)}+C\|\mathbf{g}\|_{H^{-1 / 2}(\partial \Omega)}+2 \rho_{S} \mid \dot{\theta}_{N}\left\|\partial_{t} \mathbf{d}_{N}\right\|_{L^{2}(\Omega)}\right]\right) \\
& +\left(\left[\|\mathbf{f}\|_{L^{2}(\Omega)}+\rho_{S}\left|\dot{\theta}_{N}\right|^{2}\left\|\overrightarrow{G \xi}+\mathbf{d}_{N}\right\|_{L^{2}(\Omega)}+2 \rho_{S} \mid \dot{\theta}_{N}\left\|\partial_{t} \mathbf{d}_{N}\right\|_{L^{2}(\Omega)}\right]\left\|\partial_{t t} \beta_{N}\right\|_{L^{2}(\Omega)}\right. \\
& \left.+\left[C\|\mathbf{g}\|_{H^{-1 / 2}(\partial \Omega)}+C\left\|\mathbf{d}_{N}\right\|_{H^{1}(\Omega)}\right]\left\|\partial_{t t} \beta_{N}\right\|_{H^{1}(\Omega)}\right),
\end{aligned}
$$

where we used:

$$
\left|\int_{\Omega} \mathbf{d}_{N} \wedge \partial_{t t} \omega_{N}\right|=\left|\int_{\Omega}\left[\left(\begin{array}{cc}
0 & -1 \\
1 & 0
\end{array}\right) \mathbf{d}_{N}\right] \cdot \partial_{t t} \omega_{N}\right| \leq\left\|\mathbf{d}_{N}\right\|_{H^{1}(\Omega)}\left\|\partial_{t t} \omega_{N}\right\|_{\left(H^{1}(\Omega)\right)^{\prime}} .
$$

We next remark that the following inverse inequality holds

$$
\left\|\partial_{t t} \beta_{N}\right\|_{H^{1}(\Omega)} \leq C \sqrt{\lambda_{N_{0}}}\left\|\partial_{t t} \beta_{N}\right\|_{L^{2}(\Omega)} .
$$


Thus by integrating $(48)$ over $(0, T)$ and thanks to the Hölder inequality the following inequality holds:

$$
\begin{aligned}
\| \ddot{\theta}_{N} & \left\|_{L^{2}(0, T)}^{2}+\right\| \partial_{t t} \beta_{N}\|\|_{L^{2}\left(0, T ; L^{2}(\Omega)\right)}^{2} \leq C\left\|\ddot{\theta}_{N}\right\|_{L^{2}(0, T)}\left\|\mathbf{d}_{N}\right\|_{L^{\infty}\left(0, T ; H^{1}(\Omega)\right)}\left\|\partial_{t t} \omega_{N}\right\|_{L^{2}\left(0, T ;\left(H^{1}(\Omega)\right)^{\prime}\right)} \\
& +C\left\|\ddot{\theta}_{N}\right\|_{L^{2}(0, T)}\left\|\overrightarrow{G \xi}+\mathbf{d}_{N}\right\|_{L^{\infty}\left(0, T ; H^{1}(\Omega)\right)}\left(\|\mathbf{f}\|_{L^{2}\left(0, T ; L^{2}(\Omega)\right)}+C\|\mathbf{g}\|_{L^{2}\left(0, T ; H^{-1 / 2}(\partial \Omega)\right)}\right. \\
& \left.+2 \rho_{S}\left\|\dot{\theta}_{N}\right\|_{L^{\infty}(0, T)}\left\|\partial_{t} \mathbf{d}_{N}\right\|_{L^{2}\left(0, T ; L^{2}(\Omega)\right)}\right)+C\left(\|\mathbf{f}\|_{L^{2}\left(0, T ; L^{2}(\Omega)\right)}\right. \\
& +\rho_{S}\left\|\dot{\theta}_{N}\right\|_{L^{4}(0, T)}^{2}\left\|\overrightarrow{G \xi}+\mathbf{d}_{N}\right\|_{L^{\infty}\left(0, T ; L^{2}(\Omega)\right)}+2 \rho_{S}\left\|\partial_{t} \mathbf{d}_{N}\right\|_{L^{\infty}\left(0, T ; L^{2}(\Omega)\right)} \\
& \left.+\left[\|\mathbf{g}\|_{L^{2}\left(0, T ; H^{-1 / 2}(\partial \Omega)\right)}+\left\|\mathbf{d}_{N}\right\|_{L^{2}\left(0, T ; H^{1}(\Omega)\right)}\right] C \sqrt{\lambda_{N_{0}}}\right)\left\|\partial_{t t} \beta_{N}\right\|_{L^{2}\left(0, T ; L^{2}(\Omega)\right)}
\end{aligned}
$$

Thanks to the energy estimates we deduce:

$$
\begin{aligned}
\left\|\ddot{\theta}_{N}\right\|_{L^{2}(0, T)}^{2}+\| & \partial_{t t} \beta_{N}\left\|_{L^{2}\left(0, T ; L^{2}(\Omega)\right)}^{2} \leq C_{N_{0}}\right\| \partial_{t t} \beta_{N} \|_{L^{2}\left(0, T ; L^{2}(\Omega)\right)} \\
& +\left\|\ddot{\theta}_{N}\right\|_{L^{2}(0, T)}\left(C\left\|\partial_{t t} \omega_{N}\right\|_{L^{2}\left(0, T ;\left(H^{1}(\Omega)\right)^{\prime}\right)}+C\right),
\end{aligned}
$$

where $C$ depends on the data (initial data, exterior forces, $\delta$ ) and $C_{N_{0}}$ depends on the data and on $\lambda_{N_{0}}$. Then using Young inequality:

$$
\left\|\ddot{\theta}_{N}\right\|_{L^{2}(0, T)}^{2}+\left\|\partial_{t t} \beta_{N}\right\|_{L^{2}\left(0, T ; L^{2}(\Omega)\right)}^{2} \leq C_{N_{0}}+C\left\|\ddot{\theta}_{N}\right\|_{L^{2}(0, T)}\left\|\partial_{t t} \omega_{N}\right\|_{L^{2}\left(0, T ;\left(H^{1}(\Omega)\right)^{\prime}\right)},
$$

where once again $C$ depends on the data (initial data, exterior forces, $\delta$ ) and $C_{N_{0}}$ depends on the data and on $\lambda_{N_{0}}$.

\section{Final estimate}

From (44) and (49) the following inequality holds:

$$
\left\|\ddot{\theta}_{N}\right\|_{L^{2}(0, T)}^{2}+\left\|\partial_{t t} \beta_{N}\right\|_{L^{2}\left(0, T ; L^{2}(\Omega)\right)}^{2} \leq C_{N_{0}}+\frac{C}{\sqrt{\lambda_{N_{0}}}}\left\|\ddot{\theta}_{N}\right\|_{L^{2}(0, T)}^{2},
$$

Thus choosing $N_{0}$ sufficently large with respect to the data and $\delta$ we obtain bounds on $\left\|\ddot{\theta}_{N}\right\|_{L^{2}(0, T)}$ and on $\left\|\partial_{t t} \beta_{N}\right\|_{L^{2}\left(0, T ; L^{2}(\Omega)\right)}$. Back into (44) it shows that $\left\|\partial_{t t} \omega_{N}\right\|_{L^{2}\left(0, T ;\left(H^{1}(\Omega)\right)^{\prime}\right)}$ is also bounded. All those bounds are independent of $N$.

To summarize, we have estimated $\ddot{\theta}_{N}$ in $L^{2}(0, T)$ and $\partial_{t t} \mathbf{d}_{N}$ in $L^{2}\left(0, T ;\left(H^{1}(\Omega)\right)^{\prime}\right)$ independently of $N$, for any $T$ such that $\mathbf{d}_{N} \in C^{0}\left([0, T] ; \mathbf{Y}_{0}^{\delta}\right)$.

\subsubsection{Passage to the limit}

In this subsection, we pass to the limit as $N \longrightarrow+\infty$ in the discrete sytem (33). We have just proven that $\left(\theta_{N}\right)_{N}$ and $\left(\mathbf{d}_{N}\right)_{N}$ are bounded independently of $N$ respectively in $H^{2}(0, T)$ and $H^{2}\left(0, T ;\left(H^{1}(\Omega)\right)^{\prime}\right) \cap W^{1, \infty}\left(0, T ; L^{2}(\Omega)\right) \cap L^{\infty}\left(0, T ; H^{1}(\Omega)\right)$.

- We rewrite the first equation of $(33)$ as follows:

$$
\frac{d}{d t}\left(J_{N} \dot{\theta}_{N}+\rho_{S} \int_{\Omega} \mathbf{d}_{N} \wedge \partial_{t} \mathbf{d}_{N}\right)=\int_{\Omega} R_{\theta_{N}}\left(\overrightarrow{G \xi}+\mathbf{d}_{N}\right) \wedge \mathbf{f}+\int_{\partial \Omega} R_{\theta_{N}}\left(\overrightarrow{G \xi}+\mathbf{d}_{N}\right) \wedge \mathbf{g} .
$$

Remind now that $\mathbf{d}_{N}$ is bounded in $W^{1, \infty}\left(0, T ; L^{2}(\Omega)\right) \cap L^{\infty}\left(0, T ; H^{1}(\Omega)\right)$, from the sequence of imbeddings:

$$
W^{1, \infty}\left(0, T ; L^{2}(\Omega)\right) \cap L^{\infty}\left(0, T ; H^{1}(\Omega)\right) \subset H^{1}((0, T) \times \Omega) \stackrel{c}{\hookrightarrow} L^{2}((0, T) \times \Omega),
$$

where the last injection is compact, we derive that there exists a subsequence ${ }^{1}\left(\mathbf{d}_{\eta}\right)_{\eta}$ of $\left(\mathbf{d}_{N}\right)_{N}$ that converges towards $\mathbf{d}$ strongly in $L^{2}\left(0, T ; L^{2}(\Omega)\right)$. Moreover $\dot{\theta}_{N}$ being bounded in $H^{1}(0, T)$, there exists a subsequence such that $\dot{\theta}_{\eta} \longrightarrow \dot{\theta}$ in $C^{0}([0, T])$. Thus :

$$
J_{\eta} \dot{\theta}_{\eta} \longrightarrow J \dot{\theta} \quad \text { in } L^{2}(0, T) .
$$

${ }^{1}$ for the sake of simplicity we will denote all the subsequences with a subscript $\eta$. 
Moreover, $\partial_{t} \mathbf{d}_{\eta} \rightarrow \partial_{t} \mathbf{d}$ weakly in $L^{2}\left(0, T ; L^{2}(\Omega)\right)$, and $\mathbf{d}_{\eta} \longrightarrow \mathbf{d}$ strongly in $L^{2}\left(0, T ; L^{2}(\Omega)\right)$, thus:

$$
\int_{\Omega} \mathbf{d}_{\eta} \wedge \partial_{t} \mathbf{d}_{\eta} \longrightarrow \int_{\Omega} \mathbf{d} \wedge \partial_{t} \mathbf{d} \quad \text { weakly in } L^{1}(0, T) .
$$

Next, $\theta_{N}$ being bounded in $H^{1}(0, T) \stackrel{c}{\hookrightarrow} C^{0}([0, T])$, we obtain that $R_{\theta_{\eta}} \longrightarrow R_{\theta}$ uniformly on $[0, T]$. The following convergences: $\mathbf{d}_{\eta} \longrightarrow \mathbf{d}$ strongly in $L^{2}\left(0, T ; L^{2}(\Omega)\right), \mathbf{d}_{\eta} \rightarrow \mathbf{d}$ weakly in $L^{2}\left(0, T ; H^{1}(\Omega)\right)$ enable us to pass to the limit in the forcing terms:

$$
\begin{array}{rlr}
\int_{\Omega} R_{\theta_{\eta}}\left(\overrightarrow{G \xi}+\mathbf{d}_{\eta}\right) \wedge \mathbf{f} & \longrightarrow \int_{\Omega} R_{\theta}(\overrightarrow{G \xi}+\mathbf{d}) \wedge \mathbf{f} & \text { strongly in } L^{2}(0, T) \\
\int_{\partial \Omega}^{\Omega} R_{\theta_{\eta}}\left(\overrightarrow{G \xi}+\mathbf{d}_{\eta}\right) \wedge \mathbf{g} & \rightarrow \int_{\partial \Omega}^{R_{\theta}(\overrightarrow{G \xi}+\mathbf{d}) \wedge \mathbf{g}} \quad \text { weakly in } L^{2}(0, T)
\end{array}
$$

Next we pass to the limit in the equation (50) to obtain:

$$
\frac{d}{d t}\left(J \dot{\theta}+\rho_{S} \int_{\Omega} \mathbf{d} \wedge \partial_{t} \mathbf{d}\right)=\int_{\Omega} R_{\theta}(\overrightarrow{G \xi}+\mathbf{d}) \wedge \mathbf{f}+\int_{\partial \Omega} R_{\theta}(\overrightarrow{G \xi}+\mathbf{d}) \wedge \mathbf{g} \quad \text { in } \mathcal{D}^{\prime}(0, T)
$$

Due to the regularities of the solution the equality (51) takes place in $L^{2}(0, T)$.

- For $1 \leq i \leq N$ fixed, we pass to the limit in the remainder of the discrete system (33). Since $\mathbf{d}_{N}$ is bounded in $H^{2}\left(0, T ;\left(H^{1}(\Omega)\right)^{\prime}\right) \cap L^{\infty}\left(0, T ; H^{1}(\Omega)\right)$, the linear terms are easy to deal with:

$$
\begin{array}{ll}
\int_{\Omega} \partial_{t t} \mathbf{d}_{\eta} \cdot \psi_{i} & \rightarrow \int_{\Omega} \partial_{t t} \mathbf{d} \cdot \psi_{i}
\end{array} \quad \text { weakly in } L^{2}(0, T) .
$$

Next since $\dot{\theta}_{\eta} \longrightarrow \dot{\theta}$ in $C^{0}([0, T])$ and $\partial_{t} \mathbf{d}_{\eta} \rightarrow \partial_{t} \mathbf{d}$ weakly in $L^{2}\left(0, T ; L^{2}(\Omega)\right)$ we have:

$$
\dot{\theta}_{\eta} \int_{\Omega} \partial_{t} \mathbf{d}_{\eta} \wedge \psi_{i} \quad \rightarrow \quad \dot{\theta} \int_{\Omega} \partial_{t} \mathbf{d} \wedge \psi_{i} \quad \text { weakly in } L^{2}(0, T)
$$

Moreover $\ddot{\theta}_{N}$ is bounded in $L^{2}(0, T)$, then $\ddot{\theta}_{\eta} \rightarrow \ddot{\theta}$ weakly in $L^{2}(0, T)$; then reminding that $\mathbf{d}_{\eta} \longrightarrow \mathbf{d}$ strongly in $L^{2}\left(0, T ; L^{2}(\Omega)\right.$ ) (for instance) we obtain:

$$
\ddot{\theta}_{\eta} \int_{\Omega} \mathbf{d}_{\eta} \wedge \psi_{i} \rightarrow \ddot{\theta} \int_{\Omega} \mathbf{d} \wedge \psi_{i} \quad \text { weakly in } L^{1}(0, T) .
$$

Similarly, since $\dot{\theta}_{\eta} \longrightarrow \dot{\theta}$ in $C^{0}([0, T])$ and $\mathbf{d}_{\eta} \longrightarrow \mathbf{d}$ strongly in $L^{2}\left(0, T ; L^{2}(\Omega)\right)$ then:

$$
\left(\dot{\theta}_{\eta}\right)^{2} \int_{\Omega}\left(\overrightarrow{G \xi}+\mathbf{d}_{\eta}\right) \cdot \psi_{i} \quad \longrightarrow \quad(\dot{\theta})^{2} \int_{\Omega}(\overrightarrow{G \xi}+\mathbf{d}) \cdot \psi_{i} \quad \text { strongly in } L^{2}(0, T) .
$$

Finally as previously the forcing terms give:

$$
\begin{aligned}
\int_{\Omega} R_{\theta_{\eta}} \psi_{i} \cdot \mathbf{f} & \longrightarrow \int_{\Omega} R_{\theta} \psi_{i} \cdot \mathbf{f} \\
\int_{\partial \Omega} R_{\theta_{\eta}} \psi_{i} \cdot \mathbf{g} & \longrightarrow \int_{\partial \Omega} R_{\theta} \psi_{i} \cdot \mathbf{g}
\end{aligned} \quad \text { in } C^{0}([0, T])
$$

Consequently for any given $i$, we have:

$$
\begin{aligned}
\rho_{S}\left(\int_{\Omega} \partial_{t t} \mathbf{d} \cdot \psi_{i}+2 \dot{\theta} \int_{\Omega} \partial_{t} \mathbf{d}\right. & \left.\wedge \psi_{i}+\ddot{\theta} \int_{\Omega} \mathbf{d} \wedge \psi_{i}-(\dot{\theta})^{2} \int_{\Omega}(\overrightarrow{G \xi}+\mathbf{d}) \cdot \psi_{i}\right) \\
& +\int_{\Omega} \bar{\sigma}(\mathbf{d}): \nabla \psi_{i}=\int_{\Omega} \mathbf{f} \cdot R_{\theta} \psi_{i}+\int_{\partial \Omega} \mathbf{g} \cdot R_{\theta} \psi_{i} \quad \text { in } L^{1}(0, T)
\end{aligned}
$$


Since $\mathcal{E}_{1}=<\psi_{i}>_{i \geq 1}$, the equation is satisfied for any function $\mathbf{b} \in \mathcal{E}_{1}$. Moreover, due to the regularities of the solution the equality (52) takes place actually in $L^{2}(0, T)$. We next check that $\mathbf{d} \in C^{0}\left([0, T] ; \mathbf{Y}_{0}^{\delta}\right)$. Indeed $\mathbf{d}_{N} \in C^{0}\left([0, T] ; \mathbf{Y}_{0}^{\delta}\right)$, thus for all $t \in[0, T], \int_{\Omega} \mathbf{d}_{N}=0, \int_{\Omega} \mathbf{d}_{N} \wedge \overrightarrow{G \xi}=0$ and $\int_{\Omega}\left(\overrightarrow{G \xi}+\mathbf{d}_{N}\right) \cdot \overrightarrow{G \xi} \geq \delta>0$. Since $\mathbf{d}_{N} \longrightarrow \mathbf{d}$ strongly in $C^{0}\left([0, T] ; L^{2}(\Omega)\right)$ we have:

$$
\forall t \in[0, T], \quad \int_{\Omega} \mathbf{d}=0, \int_{\Omega} \mathbf{d} \wedge \overrightarrow{G \xi}=0 \text { and } \int_{\Omega}(\overrightarrow{G \xi}+\mathbf{d}) \cdot \overrightarrow{G \xi} \geq \delta>0,
$$

which state that $\mathbf{d} \in C^{0}\left([0, T] ; \mathbf{Y}_{0}^{\delta}\right)$.

We next verify that the initial conditions are satisfied. The angle $\theta_{N}$ is bounded in $H^{2}(0, T)$ which is compactly embeded into $C^{1}([0, T])$, then $\theta_{\eta}(0)=\theta_{0} \longrightarrow \theta(0)$ and $\dot{\theta}_{\eta}(0)=\dot{\theta}_{1} \longrightarrow \dot{\theta}(0)$. Similarly, the fact that $\mathbf{d}_{N}$ is bounded in $H^{2}\left(0, T ;\left(H^{1}(\Omega)\right)^{\prime}\right) \cap W^{1, \infty}\left(0, T ; L^{2}(\Omega)\right)$, implies that (cf [11], theorem 3. 1 p. 23) $\mathbf{d}_{N} \in C^{0}\left([0, T] ; L^{2}(\Omega)\right)$ and $\partial_{t} \mathbf{d}_{N} \in C^{0}\left([0, T] ;\left(H^{1}(\Omega)\right)^{\prime}\right)$, and that $\mathbf{d}_{\eta}(0) \longrightarrow \mathbf{d}(0)$ in $L^{2}(\Omega), \partial_{t} \mathbf{d}_{\eta}(0) \longrightarrow \partial_{t} \mathbf{d}(0)$ in $\left(H^{1}(\Omega)\right)^{\prime}$. But from $(34)$, we have $\mathbf{d}_{\eta}(0)=$ $\mathbf{d}_{\eta 0} \longrightarrow \mathbf{d}_{0}$ in $H^{1}(\Omega)$ and $\partial_{t} \mathbf{d}_{\eta}(0)=\mathbf{d}_{\eta 1} \longrightarrow \mathbf{d}_{1}$ in $L^{2}(\Omega)$. Consequently we obtain $\mathbf{d}(0)=\mathbf{d}_{0}$ in $H^{1}(\Omega)$ and $\partial_{t} \mathbf{d}(0)=\mathbf{d}_{1}$ in $L^{2}(\Omega)$.

Finally since $\mathbf{d} \in H^{2}\left(0, T ;\left(H^{1}(\Omega)\right)^{\prime}\right) \cap W^{1, \infty}\left(0, T ; L^{2}(\Omega)\right)$, we deduce some additional regularities. We have (see $[11]$ lemme 8.2 p. 298) that $\mathbf{d} \in C^{0}\left([0, T] ; H^{1}(\Omega)_{w}\right)$ and $\partial_{t} \mathbf{d} \in C^{0}\left([0, T] ; L^{2}(\Omega)_{w}\right)$, where $H^{1}(\Omega)_{w}\left(\operatorname{resp} . L^{2}(\Omega)_{w}\right.$.) denotes the space $H^{1}(\Omega)$ (resp. $L^{2}(\Omega)$ ) endowed with its weak topology.

Thus we have proven the existence of a local solution of (30) for any data. In the next section we prove that this solution is unique.

\subsection{Uniqueness of the solution}

We suppose that there exists two solutions $\left(\theta_{i}, \mathbf{d}_{i}\right), i=1,2$ of the system (30) (the uniqueness of the translation is straightfoward). We denote by the subscript $i$ the quantities associated with $\left(\theta_{i}, \mathbf{d}_{i}\right)$, like the rotation $R_{i}=R_{\theta_{i}}$. We recall the system satisfied by the solutions:

$$
\left\{\begin{array}{cc}
\frac{d}{d t}\left(J_{i} \dot{\theta}_{i}\right)+\rho_{S} \int_{\Omega} \mathbf{d}_{i} \wedge \partial_{t t} \mathbf{d}_{i}=\int_{\Omega} R_{i}\left(\overrightarrow{G \xi}+\mathbf{d}_{i}\right) \wedge \mathbf{f}+\int_{\partial \Omega} R_{i}\left(\overrightarrow{G \xi}+\mathbf{d}_{i}\right) \wedge \mathbf{g} & \text { in } L^{2}(0, T) \\
\forall \mathbf{b} \in \mathcal{E}_{1}, \quad \rho_{S}\left(\int_{\Omega} \partial_{t t} \mathbf{d}_{i} \cdot \mathbf{b}+2 \dot{\theta}_{i} \int_{\Omega} \partial_{t} \mathbf{d}_{i} \wedge \mathbf{b}+\ddot{\theta}_{i} \int_{\Omega} \mathbf{d}_{i} \wedge \mathbf{b}-\left(\dot{\theta}_{i}\right)^{2} \int_{\Omega}\left(\overrightarrow{G \xi}+\mathbf{d}_{i}\right) \cdot \mathbf{b}\right) & \\
+\int_{\Omega} \bar{\sigma}\left(\mathbf{d}_{i}\right): \nabla \mathbf{b}=\int_{\Omega} \mathbf{f} \cdot R_{i} \mathbf{b}+\int_{\partial \Omega} \mathbf{g} \cdot R_{i} \mathbf{b} & \text { in } L^{2}(0, T)
\end{array}\right.
$$

where we denoted by $J_{i}=\rho_{S} \int_{\Omega}\left(\overrightarrow{G \xi}+\mathbf{d}_{i}\right)^{2}$ the inertia momentum associated with $\mathbf{d}_{i}$. To prove the uniqueness of the rotation angle and of the displacement $\mathbf{d}$, we divide our proof in two steps. The first step is done in a formal way assuming that $\partial_{t} \mathbf{d}$ belongs to $L^{\infty}\left(0, T ; \mathcal{E}_{1}\right)$ and consequently is an admissible test function. This step consists in algebraic calculations so that we rewrite the system as an equality (see (58)) in terms of $\theta=\theta_{2}-\theta_{1}$ and $\mathbf{d}=\mathbf{d}_{2}-\mathbf{d}_{1}$. We then estimate the terms on the right hand side of this equality. Finally we apply the Gronwall lemma to conclude (for the regularized case). The second step adressess the general case (where $\partial_{t} \mathbf{d}$ only belongs to $\left.\mathrm{E}^{\infty}\left(0, T ; L^{2}(\Omega)\right)\right)$ thanks to a regularization argument.

\section{Step 1.}

On one hand, we subtract the equations concerning the angles $\theta_{2}$ and $\theta_{1}$, and we multiply the new equation by $\dot{\theta}$ to get:

$$
\begin{aligned}
\dot{\theta} \frac{d}{d t}\left(\dot{\theta}_{2} J_{2}-\dot{\theta}_{1} J_{1}\right)+\rho_{S} \dot{\theta} \int_{\Omega}\left(\mathbf{d}_{2} \wedge \partial_{t t} \mathbf{d}_{2}-\mathbf{d}_{1}\right. & \left.\wedge \partial_{t t} \mathbf{d}_{1}\right) \\
=\dot{\theta} \int_{\Omega}\left[R_{2}\left(\overrightarrow{G \xi}+\mathbf{d}_{2}\right)-R_{1}\left(\overrightarrow{G \xi}+\mathbf{d}_{1}\right)\right] & \wedge \mathbf{f}+\dot{\theta} \int_{\partial \Omega}\left[R_{2}\left(\overrightarrow{G \xi}+\mathbf{d}_{2}\right)-R_{1}\left(\overrightarrow{G \xi}+\mathbf{d}_{1}\right)\right] \wedge \mathbf{g} .
\end{aligned}
$$


Then, by making $\mathbf{d}$ and $\theta$ appear, we have:

$$
\begin{aligned}
& \dot{\theta} \frac{d}{d t}\left(\dot{\theta} J_{1}+\dot{\theta}_{2}\left(J_{2}-J_{1}\right)\right)+\rho_{S} \dot{\theta} \int_{\Omega} \mathbf{d}_{1} \wedge \partial_{t t} \mathbf{d}+\rho_{S} \dot{\theta} \int_{\Omega} \mathbf{d} \wedge \partial_{t t} \mathbf{d}_{2}= \\
& \int_{\Omega}\left[\left(R_{2}-R_{1}\right)\left(\overrightarrow{G \xi}+\mathbf{d}_{2}\right)+R_{1} \mathbf{d}\right] \wedge \mathbf{f}+\dot{\theta} \int_{\partial \Omega}\left[\left(R_{2}-R_{1}\right)\left(\overrightarrow{G \xi}+\mathbf{d}_{2}\right)+R_{1} \mathbf{d}\right] \wedge \mathbf{g} .
\end{aligned}
$$

On the other hand, we subtract the equations concerning the perturbations $\mathbf{d}_{2}$ and $\mathbf{d}_{1}$, and we have for all $\mathbf{b} \in \mathcal{E}_{1}$ :

$$
\begin{aligned}
& \rho_{S}\left(\int_{\Omega} \partial_{t t} \mathbf{d} \cdot \mathbf{b}+2 \dot{\theta} \int_{\Omega} \partial_{t} \mathbf{d}_{1} \wedge \mathbf{b}+2 \dot{\theta}_{2} \int_{\Omega} \partial_{t} \mathbf{d} \wedge \mathbf{b}+\ddot{\theta} \int_{\Omega} \mathbf{d}_{1} \wedge \mathbf{b}+\ddot{\theta}_{2} \int_{\Omega} \mathbf{d} \wedge \mathbf{b}\right. \\
& \left.+\mathcal{T}\left(\dot{\theta}_{1}, \mathbf{d}_{1}, \mathbf{b}\right)-\mathcal{T}\left(\dot{\theta}_{2}, \mathbf{d}_{2}, \mathbf{b}\right)\right)+\int_{\Omega} \bar{\sigma}(\mathbf{d}): \nabla \mathbf{b}=\int_{\Omega} \mathbf{f} \cdot\left(R_{2}-R_{1}\right) \mathbf{b}+\int_{\partial \Omega} \mathbf{g} \cdot\left(R_{2}-R_{1}\right) \mathbf{b},
\end{aligned}
$$

where $\mathcal{T}$ is defined by $\mathcal{T}(\theta, \mathbf{d}, \mathbf{b})=(\theta)^{2} \int_{\Omega}(\overrightarrow{G \xi}+\mathbf{d}) \cdot \mathbf{b}$.

Let us assume in this step only that $\partial_{t} \mathbf{d} \in L^{\infty}\left(0, T ; \mathcal{E}_{1}\right)$. So, it is now licit to take $\mathbf{b}=\partial_{t} \mathbf{d}$ in (54):

$$
\begin{aligned}
& \rho_{S}\left(\int_{\Omega} \partial_{t t} \mathbf{d} \cdot \partial_{t} \mathbf{d}+2 \dot{\theta} \int_{\Omega} \partial_{t} \mathbf{d}_{1} \wedge \partial_{t} \mathbf{d}+\ddot{\theta} \int_{\Omega} \mathbf{d}_{1} \wedge \partial_{t} \mathbf{d}+\ddot{\theta}_{2} \int_{\Omega} \mathbf{d} \wedge \partial_{t} \mathbf{d}\right. \\
& \left.+\mathcal{T}\left(\dot{\theta}_{1}, \mathbf{d}_{1}, \partial_{t} \mathbf{d}\right)-\mathcal{T}\left(\dot{\theta}_{2}, \mathbf{d}_{2}, \partial_{t} \mathbf{d}\right)\right)+\left(\left(\mathbf{d}, \partial_{t} \mathbf{d}\right)\right)=\int_{\Omega} \mathbf{f} \cdot\left(R_{2}-R_{1}\right) \partial_{t} \mathbf{d}+\int_{\partial \Omega} \mathbf{g} \cdot\left(R_{2}-R_{1}\right) \partial_{t} \mathbf{d} .
\end{aligned}
$$

We now set $\mathcal{F}=\mathcal{F}_{\mathbf{f}}+\mathcal{F}_{\mathbf{g}}$, which contains the terms of the external forces (coming from (53) and (55)):

$$
\begin{aligned}
& \mathcal{F}_{\mathbf{f}}=\dot{\theta} \int_{\Omega}\left[\left(R_{2}-R_{1}\right)\left(\overrightarrow{G \xi}+\mathbf{d}_{2}\right)+R_{1} \mathbf{d}\right] \wedge \mathbf{f}+\int_{\Omega} \mathbf{f} \cdot\left(R_{2}-R_{1}\right) \partial_{t} \mathbf{d} \\
& \mathcal{F}_{\mathbf{g}}=\dot{\theta} \int_{\partial \Omega}\left[\left(R_{2}-R_{1}\right)\left(\overrightarrow{G \xi}+\mathbf{d}_{2}\right)+R_{1} \mathbf{d}\right] \wedge \mathbf{g}+\int_{\partial \Omega} \mathbf{g} \cdot\left(R_{2}-R_{1}\right) \partial_{t} \mathbf{d} .
\end{aligned}
$$

Thus, by adding each side of (53) and (55), we obtain:

$$
\begin{aligned}
{\left[\dot{\theta} \frac{d}{d t}\left(\dot{\theta} J_{1}\right)+\rho_{S} \dot{\theta} \int_{\Omega} \mathbf{d}_{1} \wedge \partial_{t t} \mathbf{d}+\rho_{S} \int_{\Omega} \partial_{t t} \mathbf{d} \cdot \partial_{t} \mathbf{d}+\rho_{S} \dot{\theta} \int_{\Omega} \partial_{t} \mathbf{d}_{1} \wedge \partial_{t} \mathbf{d}+\rho_{S} \ddot{\theta} \int_{\Omega} \mathbf{d}_{1} \wedge \partial_{t} \mathbf{d}\right] } \\
+\dot{\theta} \frac{d}{d t}\left(\dot{\theta}_{2}\left(J_{2}-J_{1}\right)\right)+\rho_{S} \dot{\theta} \int_{\Omega} \mathbf{d} \wedge \partial_{t t} \mathbf{d}_{2}+\rho_{S} \dot{\theta} \int_{\Omega} \partial_{t} \mathbf{d}_{1} \wedge \partial_{t} \mathbf{d}+\rho_{S} \ddot{\theta}_{2} \int_{\Omega} \mathbf{d} \wedge \partial_{t} \mathbf{d} \\
+\rho_{S}\left(\mathcal{T}\left(\dot{\theta}_{1}, \mathbf{d}_{1}, \partial_{t} \mathbf{d}\right)-\mathcal{T}\left(\dot{\theta}_{2}, \mathbf{d}_{2}, \partial_{t} \mathbf{d}\right)\right)+\frac{1}{2} \frac{d}{d t}((\mathbf{d}, \mathbf{d}))=\mathcal{F}
\end{aligned}
$$

Let us set

$$
y(t)=\rho_{S}\left\|\dot{\theta} \overrightarrow{e_{z}} \wedge R_{1}\left(\overrightarrow{G \xi}+\mathbf{d}_{1}\right)+R_{1} \partial_{t} \mathbf{d}\right\|_{L^{2}(\Omega)}^{2}=|\dot{\theta}|^{2} J_{1}+2 \rho_{S} \dot{\theta} \int_{\Omega} \mathbf{d}_{1} \wedge \partial_{t} \mathbf{d}+\rho_{S} \int_{\Omega}\left|\partial_{t} \mathbf{d}\right|^{2},
$$

and remark that:

$$
\frac{\dot{y}(t)}{2}=\ddot{\theta} \dot{\theta} J_{1}+|\dot{\theta}|^{2} \frac{\dot{J}_{1}}{2}+\rho_{S} \ddot{\theta} \int_{\Omega} \mathbf{d}_{1} \wedge \partial_{t} \mathbf{d}+\rho_{S} \dot{\theta} \int_{\Omega} \partial_{t} \mathbf{d}_{1} \wedge \partial_{t} \mathbf{d}+\rho_{S} \dot{\theta} \int_{\Omega} \mathbf{d}_{1} \wedge \partial_{t t} \mathbf{d}+\rho_{S} \int_{\Omega} \partial_{t} \mathbf{d} \cdot \partial_{t t} \mathbf{d} .
$$

Consequently, the terms of the first line of (56) between the square brackets are equal to $\frac{\dot{y}(t)}{2}+$ $(\dot{\theta})^{2} \frac{\dot{J}_{1}}{2}$. Thus we deduce that:

$$
\frac{\dot{y}(t)}{2}+\frac{1}{2} \frac{d}{d t}((\mathbf{d}, \mathbf{d}))=\mathcal{F}-\mathcal{R},
$$


where:

$$
\begin{aligned}
\mathcal{R}=(\dot{\theta})^{2} \frac{\dot{J}_{1}}{2}+\dot{\theta} & \frac{d}{d t} \\
& \left(\dot{\theta}_{2}\left(J_{2}-J_{1}\right)\right)+\rho_{S} \dot{\theta} \int_{\Omega} \mathbf{d} \wedge \partial_{t t} \mathbf{d}_{2} \\
& +\rho_{S} \dot{\theta} \int_{\Omega} \partial_{t} \mathbf{d}_{1} \wedge \partial_{t} \mathbf{d}+\rho_{S} \ddot{\theta}_{2} \int_{\Omega} \mathbf{d} \wedge \partial_{t} \mathbf{d}+\rho_{S}\left(\mathcal{T}\left(\dot{\theta}_{1}, \mathbf{d}_{1}, \partial_{t} \mathbf{d}\right)-\mathcal{T}\left(\dot{\theta}_{2}, \mathbf{d}_{2}, \partial_{t} \mathbf{d}\right)\right)
\end{aligned}
$$

Furthermore, we rewrite $\mathcal{T}\left(\dot{\theta}_{1}, \mathbf{d}_{1}, \partial_{t} \mathbf{d}\right)-\mathcal{T}\left(\dot{\theta}_{2}, \mathbf{d}_{2}, \partial_{t} \mathbf{d}\right)$ using the identity:

$$
\begin{aligned}
a_{1}^{2} b_{1}-a_{2}^{2} b_{2} & =a_{1} b_{1}\left(a_{1}-a_{2}\right)+a_{1} a_{2} b_{1}-a_{2} b_{2}\left(a_{2}-a_{1}\right)-a_{2} b_{2} a_{1} \\
& =-\left(a_{2}-a_{1}\right)\left(a_{1} b_{1}+a_{2} b_{2}\right)-a_{1} a_{2}\left(b_{2}-b_{1}\right) .
\end{aligned}
$$

It gives

$$
\begin{aligned}
\mathcal{T}\left(\dot{\theta}_{1}, \mathbf{d}_{1}, \partial_{t} \mathbf{d}\right)-\mathcal{T}\left(\dot{\theta}_{2}, \mathbf{d}_{2}, \partial_{t} \mathbf{d}\right) & =\left(\dot{\theta}_{1}\right)^{2} \int_{\Omega}\left(\overrightarrow{G \xi}+\mathbf{d}_{1}\right) \cdot \partial_{t} \mathbf{d}-\left(\dot{\theta}_{2}\right)^{2} \int_{\Omega}\left(\overrightarrow{G \xi}+\mathbf{d}_{2}\right) \cdot \partial_{t} \mathbf{d} \\
& =-\dot{\theta} \int_{\Omega}\left[\dot{\theta}_{1}\left(\overrightarrow{G \xi}+\mathbf{d}_{1}\right)+\dot{\theta}_{2}\left(\overrightarrow{G \xi}+\mathbf{d}_{2}\right)\right] \cdot \partial_{t} \mathbf{d}-\dot{\theta}_{1} \dot{\theta}_{2} \int_{\Omega} \mathbf{d} \cdot \partial_{t} \mathbf{d} .
\end{aligned}
$$

So $\mathcal{R}$ can be rewritten as follows:

$$
\begin{aligned}
\mathcal{R}=(\dot{\theta})^{2} & \frac{\dot{J}_{1}}{2}+\dot{\theta} \ddot{\theta}_{2}\left(J_{2}-J_{1}\right)+\dot{\theta} \dot{\theta}_{2}\left(\dot{J}_{2}-\dot{J}_{1}\right)+\rho_{S} \dot{\theta} \int_{\Omega} \mathbf{d} \wedge \partial_{t t} \mathbf{d}_{2}+\rho_{S} \dot{\theta} \int_{\Omega} \partial_{t} \mathbf{d}_{1} \wedge \partial_{t} \mathbf{d} \\
& +\rho_{S} \ddot{\theta}_{2} \int_{\Omega} \mathbf{d} \wedge \partial_{t} \mathbf{d}-\rho_{S} \dot{\theta} \int_{\Omega}\left[\dot{\theta}_{1}\left(\overrightarrow{G \xi}+\mathbf{d}_{1}\right)+\dot{\theta}_{2}\left(\overrightarrow{G \xi}+\mathbf{d}_{2}\right)\right] \cdot \partial_{t} \mathbf{d}-\rho_{S} \dot{\theta}_{1} \dot{\theta}_{2} \int_{\Omega} \mathbf{d} \cdot \partial_{t} \mathbf{d} .
\end{aligned}
$$

Now that we have rewritten the energy equality satisfied by $\mathbf{d}$ and $\theta$ we estimate $\mathcal{F}$ and $\mathcal{R}$.

- Estimate of $\mathcal{R}$.

We first begin with the terms that do not involve $\ddot{\theta}_{2}$ nor $\partial_{t t} \mathbf{d}_{2}$. We have:

$$
\begin{aligned}
\left|\frac{(\dot{\theta})^{2} \dot{J}_{1}}{2}\right| & =\rho_{S}|\dot{\theta}|^{2}\left|\int_{\Omega}\left(\overrightarrow{G \xi}+\mathbf{d}_{1}\right) \cdot \partial_{t} \mathbf{d}_{1}\right| \\
& \leq \rho_{S}\left\|\overrightarrow{G \xi}+\mathbf{d}_{1}\right\|_{L^{\infty}\left(0, T ; L^{2}(\Omega)\right)}|| \partial_{t} \mathbf{d}_{1} \|_{L^{\infty}\left(0, T ; L^{2}(\Omega)\right)}|\dot{\theta}|^{2} \leq C|\dot{\theta}|^{2},
\end{aligned}
$$

with a constant $C$ independent of time because $\mathbf{d}_{1} \in W^{1, \infty}\left(0, T ; L^{2}(\Omega)\right)$. For the same reason, we get:

$$
\left|\dot{\theta} \int_{\Omega} \partial_{t} \mathbf{d}_{1} \wedge \partial_{t} \mathbf{d}\right| \leq|\dot{\theta}|\left\|\partial_{t} \mathbf{d}_{1}\right\|_{L^{2}(\Omega)}\left\|\partial_{t} \mathbf{d}\right\|_{L^{2}(\Omega)} \leq C \mid \dot{\theta}\left\|\partial_{t} \mathbf{d}\right\|_{L^{2}(\Omega)} .
$$

Reminding that $\dot{\theta}_{2} \in L^{\infty}(0, T)$ and $\partial_{t} \mathbf{d}_{2} \in L^{\infty}\left(0, T ; L^{2}(\Omega)\right)$, we get:

$$
\left|\dot{\theta} \dot{\theta}_{2}\left(\dot{J}_{2}-\dot{J}_{1}\right)\right|=2 \rho_{S}|\dot{\theta}|\left|\dot{\theta}_{2}\right|\left|\int_{\Omega} \mathbf{d} \cdot \partial_{t} \mathbf{d}_{2}+\int_{\Omega}\left(\overrightarrow{G \xi}+\mathbf{d}_{1}\right) \cdot \partial_{t} \mathbf{d}\right| \leq C|\dot{\theta}|\left(\|\mathbf{d}\|_{L^{2}(\Omega)}+\left\|\partial_{t} \mathbf{d}\right\|_{L^{2}(\Omega)}\right) .
$$

Furthermore, since $\mathbf{d}_{1}, \mathbf{d}_{2} \in L^{\infty}\left(0, T ; L^{2}(\Omega)\right)$ and $\dot{\theta}_{1}, \dot{\theta}_{2} \in L^{\infty}(0, T)$, we have as well:

$$
\left|\dot{\theta} \int_{\Omega}\left[\dot{\theta}_{1}\left(\overrightarrow{G \xi}+\mathbf{d}_{1}\right)+\dot{\theta}_{2}\left(\overrightarrow{G \xi}+\mathbf{d}_{2}\right)\right] \cdot \partial_{t} \mathbf{d}\right| \leq C|\dot{\theta}|\left\|\partial_{t} \mathbf{d}\right\|_{L^{2}(\Omega)},
$$

and:

$$
\left|\dot{\theta}_{1} \dot{\theta}_{2} \int_{\Omega} \mathbf{d} \cdot \partial_{t} \mathbf{d}\right| \leq C\|\mathbf{d}\|_{L^{2}(\Omega)}\left\|\partial_{t} \mathbf{d}\right\|_{L^{2}(\Omega)} .
$$

We now treat the terms that require regularity on the second order derivatives with respect to time:

$$
\left|\ddot{\theta}_{2}\left(J_{2}-J_{1}\right)\right|=\rho_{S}|\dot{\theta}|\left|\ddot{\theta}_{2}\right|\left|\int_{\Omega}\left(2 \overrightarrow{G \xi}+\mathbf{d}_{2}+\mathbf{d}_{1}\right) \cdot \mathbf{d}\right| \leq C_{1}(t)|\dot{\theta}|\|\mathbf{d}\|_{L^{2}(\Omega)}
$$


with $C_{1}(\cdot) \in L^{2}(0, T)$ because $\ddot{\theta}_{2} \in L^{2}(0, T)$. For the same reason, it comes:

$$
\left|\ddot{\theta}_{2} \int_{\Omega} \mathbf{d} \wedge \partial_{t} \mathbf{d}\right| \leq\left|\ddot{\theta}_{2}\right| \mid \mathbf{d}\left\|_{L^{2}(\Omega)}\right\| \partial_{t} \mathbf{d}\left\|_{L^{2}(\Omega)} \leq C_{1}(t)\right\| \mathbf{d}\left\|_{L^{2}(\Omega)}\right\| \partial_{t} \mathbf{d} \|_{L^{2}(\Omega)} .
$$

Next the last term of $\mathcal{R}$ gives:

$$
\left|\dot{\theta} \int_{\Omega} \mathbf{d} \wedge \partial_{t t} \mathbf{d}_{2}\right| \leq|\dot{\theta}||| \partial_{t t} \mathbf{d}_{2}\left\|_{\left(\mathcal{E}_{1}\right)^{\prime}}\right\| \mathbf{d}\left\|_{H^{1}(\Omega)} \leq C_{2}(t)|\dot{\theta}|\right\| \mathbf{d} \|_{H^{1}(\Omega)} .
$$

with $C_{2}(\cdot) \in L^{2}(0, T)$ because $\partial_{t t} \mathbf{d}_{2} \in L^{2}\left(0, T ;\left(\mathcal{E}_{1}\right)^{\prime}\right)$. For the term $\mathcal{R}$, we finally find the following estimate:

$$
\begin{aligned}
\mathcal{R} \leq & C\left[|\dot{\theta}|^{2}+|\dot{\theta}|\left(\left\|\partial_{t} \mathbf{d}\right\|_{L^{2}(\Omega)}+\|\mathbf{d}\|_{L^{2}(\Omega)}\right)+\|\mathbf{d}\|_{L^{2}(\Omega)}\left\|\partial_{t} \mathbf{d}\right\|_{L^{2}(\Omega)}\right] \\
& +C_{1}(t)\|\mathbf{d}\|_{L^{2}(\Omega)}\left(|\dot{\theta}|+\left\|\partial_{t} \mathbf{d}\right\|_{L^{2}(\Omega)}\right)+C_{2}(t) \mid \dot{\theta}\|\mathbf{d}\|_{H^{1}(\Omega)} \\
\leq & C(t)\left[|\dot{\theta}|^{2}+\left\|\partial_{t} \mathbf{d}\right\|_{L^{2}(\Omega)}^{2}+\|\mathbf{d}\|_{H^{1}(\Omega)}^{2}\right] \text { thanks to Young's inequality } \\
\leq & C(t)\left[|\dot{\theta}|^{2}+\left\|\partial_{t} \mathbf{d}\right\|_{L^{2}(\Omega)}^{2}+C\|\| \mathbf{d} \|\left.\right|^{2}\right]
\end{aligned}
$$

with still $C(\cdot) \in L^{2}(0, T)$.

- Estimates of $\mathcal{F}$.

The terms where the bulk forces appears can be estimated by:

$$
\begin{aligned}
\left|\mathcal{F}_{\mathbf{f}}\right| & \leq\left|\dot{\theta} \int_{\Omega}\left[\left(R_{2}-R_{1}\right)\left(\overrightarrow{G \xi}+\mathbf{d}_{2}\right)+R_{1} \mathbf{d}\right] \wedge \mathbf{f}\right|+\left|\int_{\Omega} \mathbf{f} \cdot\left(R_{2}-R_{1}\right) \partial_{t} \mathbf{d}\right| \\
& \left.\leq\|\mathbf{f}\|_{L^{2}(\Omega)}\left[C|\dot{\theta}|\left(\left|R_{2}-R_{1}\right|+\|\mathbf{d}\|_{L^{2}(\Omega)}\right)+\left|R_{2}-R_{1}\right|\left\|\partial_{t} \mathbf{d}\right\|_{L^{2}(\Omega)}\right)\right]
\end{aligned}
$$

because $\mathbf{d}_{2} \in L^{\infty}\left(0, T ; L^{2}(\Omega)\right)$. Taking now into account the fact that sines and cosines are both lipschitz continuous, we have $\left|R_{2}-R_{1}\right| \leq C\left|\theta_{2}-\theta_{1}\right|$ and thus:

$$
\left.\begin{array}{rl}
\left|\mathcal{F}_{\mathbf{f}}\right| & \leq C(t)\left(\left|\dot{\theta}\left\|\theta|+| \dot{\theta}\left|\|\mathbf{d}\|_{L^{2}(\Omega)}+\right| \theta \mid\right\| \partial_{t} \mathbf{d} \|_{L^{2}(\Omega)}\right.\right. \\
& \leq C(t)\left(|\theta|^{2}+|\dot{\theta}|^{2}+\|\mathbf{d}\|_{L^{2}(\Omega)}^{2}+\left\|\partial_{t} \mathbf{d}\right\|_{L^{2}(\Omega)}^{2}\right.
\end{array}\right)
$$

with $C(\cdot) \in L^{2}(0, T)$ because $\mathbf{f} \in L^{2}\left(0, T ; L^{2}(\Omega)\right)$.

The first term in $\mathcal{F}_{\mathbf{g}}$ can be easily upperbounded as previously by:

$$
\begin{aligned}
\mid \dot{\theta} \int_{\partial \Omega}\left[\left(R_{2}\right.\right. & \left.\left.-R_{1}\right)\left(\overrightarrow{G \xi}+\mathbf{d}_{2}\right)+R_{1} \mathbf{d}\right] \wedge \mathbf{g} \mid \\
& \leq|\dot{\theta}| \mid \mathbf{g} \|_{H^{-1 / 2}(\partial \Omega)}\left(\left|R_{2}-R_{1}\right| \mid \overrightarrow{G \xi}+\mathbf{d}_{2}\left\|_{H^{1 / 2}(\partial \Omega)}+C\right\| \mathbf{d} \|_{H^{1 / 2}(\partial \Omega)}\right) \\
& \leq C|\dot{\theta}|\left(\left|\theta_{2}-\theta_{1}\right|+\|\mathbf{d}\|_{H^{1}(\Omega)}\right) \\
& \leq C\left(|\theta|^{2}+|\dot{\theta}|^{2}+|\|\mathbf{d}\||^{2}\right)
\end{aligned}
$$

because $\mathbf{g} \in L^{\infty}\left(0, T ; H^{-1 / 2}(\Omega)\right), \mathbf{d}_{2} \in L^{\infty}\left(0, T ; L^{2}(\Omega)\right)$. We treat the second term in $\mathcal{F}_{\mathbf{g}}$ separately ${ }^{2}$ by:

$$
\begin{aligned}
\int_{\partial \Omega} \mathbf{g} \cdot\left(R_{2}-R_{1}\right) \partial_{t} \mathbf{d} & =\frac{d}{d t}\left(\int_{\partial \Omega} \mathbf{g} \cdot\left(R_{2}-R_{1}\right) \mathbf{d}\right)-\int_{\partial \Omega} \partial_{t} \mathbf{g} \cdot\left(R_{2}-R_{1}\right) \mathbf{d} \\
& -\int_{\partial \Omega} \mathbf{g} \cdot\left(\dot{\theta}_{2} \overrightarrow{e_{z}} \wedge R_{2}-\dot{\theta}_{1} \overrightarrow{e_{z}} \wedge R_{1}\right) \mathbf{d} \\
& =\frac{d}{d t}\left(\int_{\partial \Omega} \mathbf{g} \cdot\left(R_{2}-R_{1}\right) \mathbf{d}\right)-\int_{\partial \Omega} \partial_{t} \mathbf{g} \cdot\left(R_{2}-R_{1}\right) \mathbf{d} \\
& -\int_{\partial \Omega} \mathbf{g} \cdot\left(\dot{\theta} \overrightarrow{e_{z}} \wedge R_{2}+\dot{\theta}_{1} \overrightarrow{e_{z}} \wedge\left(R_{2}-R_{1}\right)\right) \mathbf{d}
\end{aligned}
$$

${ }^{2}$ Considering $\left|\int_{\partial \Omega} \mathbf{g} \cdot\left(R_{2}-R_{1}\right) \partial_{t} \mathbf{d}\right| \leq C|\theta|\left\|\partial_{t} \mathbf{d}\right\|_{H^{1}(\Omega)}$ (because $\partial_{t} \mathbf{d} \in \mathcal{E}_{1}$ ) would bring a term of the form $\left\|\left|\partial_{t} \mathbf{d}\right|\right\| \mid$ which does not appear in the left hand side of (58). 
On one hand we have:

$$
\left|\int_{\partial \Omega} \partial_{t} \mathbf{g} \cdot\left(R_{2}-R_{1}\right) \mathbf{d}\right| \leq C|| \partial_{t} \mathbf{g}\left\|_{H^{-1 / 2}(\partial \Omega)}|\theta|\right\| \mathbf{d}\left\|_{H^{1}(\Omega)} \leq C(t)|\theta|\right\||\mathbf{d} \||
$$

with $C(\cdot) \in L^{2}(0, T)$ because $\partial_{t} \mathbf{g} \in L^{2}\left(0, T ; H^{-1 / 2}(\partial \Omega)\right)$. And on the other hand, we have:

$$
\begin{aligned}
\left|\int_{\partial \Omega} \mathbf{g} \cdot\left(\dot{\theta} \overrightarrow{e_{z}} \wedge R_{2}+\dot{\theta}_{1} \overrightarrow{e_{z}} \wedge\left(R_{2}-R_{1}\right)\right) \mathbf{d}\right| & \leq C\|\mathbf{g}\|_{H^{-1 / 2}(\partial \Omega)}(|\dot{\theta}|+|\theta|)\|\mathbf{d}\|_{H^{1}(\Omega)} \\
& \leq C\left(|\theta|^{2}+|\dot{\theta}|^{2}+\|\mathbf{d}\| \|^{2}\right)
\end{aligned}
$$

because $\dot{\theta}_{1} \in L^{\infty}(0, T)$.

Thus, for the external forces terms, we finally get:

$$
\mathcal{F} \leq C(t)\left(|\theta|^{2}+|\dot{\theta}|^{2}+\left\|\partial_{t} \mathbf{d}\right\|_{L^{2}(\Omega)}^{2}+\|\mid \mathbf{d}\| \|^{2}\right)+\frac{d}{d t}\left(\int_{\partial \Omega} \mathbf{g} \cdot\left(R_{2}-R_{1}\right) \mathbf{d}\right)
$$

with $C(\cdot) \in L^{2}(0, T)$.

- Uniqueness of a regular solution.

Thanks to (58), (60) and (61) the following estimate holds:

$$
\frac{1}{2} \frac{d}{d t}\left(y(t)+\||\mathbf{d}|\|^{2}\right) \leq C(t)\left(|\theta|^{2}+|\dot{\theta}|^{2}+\left\|\partial_{t} \mathbf{d}\right\|_{L^{2}(\Omega)}^{2}+\left\||\mathbf{d} \||^{2}\right)+\frac{d}{d t}\left(\int_{\partial \Omega} \mathbf{g} \cdot\left(R_{2}-R_{1}\right) \mathbf{d}\right) .\right.
$$

We now integrate the previous inequality in time on $(0, t) \subset(0, T)$. Using the fact that $\dot{\theta}(0)=0$, $\mathbf{d}(0)=\partial_{t} \mathbf{d}(0) \equiv 0$ and $y(0)=0$, we get:

$$
y(t)+\left\||\mathbf{d} \||^{2} \leq \int_{0}^{t} C(s)\left(|\theta(s)|^{2}+|\dot{\theta}(s)|^{2}+\left\|\partial_{t} \mathbf{d}(s)\right\|_{L^{2}(\Omega)}^{2}+\left.\|\mathbf{d}(s)\|\right|^{2}\right) \mathrm{d} s+h(t)\right.
$$

with $h(t)=\int_{\partial \Omega} \mathbf{g}(t) \cdot\left(R_{2}(t)-R_{1}(t)\right) \mathbf{d}(t)$. Furthermore, $\theta(t)=\int_{0}^{t} \dot{\theta}(s) d s$. Thus (62) becomes:

$$
\begin{aligned}
y(t)+\|\mathbf{d}\| \|^{2} & \leq \int_{0}^{t} C(s)\left(\left(\int_{0}^{s} \dot{\theta}(u) \mathrm{d} u\right)^{2}+|\dot{\theta}(s)|^{2}+\left\|\partial_{t} \mathbf{d}(s)\right\|_{L^{2}(\Omega)}^{2}+\|\mathbf{d}(s)\| \|^{2}\right) \mathrm{d} s+h(t) \\
& \leq C \int_{0}^{t}(\dot{\theta}(u))^{2} \mathrm{~d} u+\int_{0}^{t} C(s)\left(|\dot{\theta}(s)|^{2}+\left\|\partial_{t} \mathbf{d}(s)\right\|_{L^{2}(\Omega)}^{2}+\|\mathbf{d}(s)\|_{\mathcal{E}_{1}}^{2}\right) \mathrm{d} s+h(t) .
\end{aligned}
$$

For the function $h$, we have:

$$
|h(t)| \leq C\|\mathbf{g}\|_{H^{-1 / 2}(\partial \Omega)}|\theta|\left\|\left|\mathbf{d}\left\|\mid \leq C\left(\int_{0}^{t}(\dot{\theta}(u))^{2} \mathrm{~d} u\right)^{\frac{1}{2}}\right\| \mathbf{d}(t)\|\|,\right.\right.
$$

with $C$ depending on the data and more particularly on $\|\mathbf{g}\| \|_{L^{\infty}\left(0, T ; H^{-1 / 2}(\partial \Omega)\right)}$.

Since $\mathbf{d}_{1} \in C^{0}\left([0, T] ; \mathbf{Y}_{0}^{\delta}\right)$, we have by (8), and (9) with $\mathbf{d}$ replaced by $\mathbf{d}_{1}$ that there exists $C>0$ such that:

$$
\forall \beta \in \mathbb{R}, \forall \mathbf{b} \in \mathcal{E}_{0}, \quad|\beta|^{2}+\|\mathbf{b}\|_{L^{2}(\Omega)}^{2} \leq \rho_{S} C\left\|\beta \overrightarrow{e_{z}} \wedge R_{1}\left(\overrightarrow{G \xi}+\mathbf{d}_{1}\right)+R_{1} \mathbf{b}\right\|_{L^{2}(\Omega)}^{2}
$$

Thus

$$
|\dot{\theta}(t)|^{2}+\left\|\partial_{t} \mathbf{d}(t)\right\|_{L^{2}(\Omega)}^{2} \leq C y(t) .
$$

The inequality (63) then becomes (taking into account (64) and using Young inequality):

$$
\begin{aligned}
& |\dot{\theta}(t)|^{2}+\left\|\partial_{t} \mathbf{d}(t)\right\|_{L^{2}(\Omega)}^{2}+\|\mathbf{d}(t)\|_{\mathcal{E}_{1}}^{2} \\
\leq & C \int_{0}^{t}(\dot{\theta}(u))^{2} \mathrm{~d} u+C \int_{0}^{t} C(s)\left(|\dot{\theta}(s)|^{2}+\left\|\partial_{t} \mathbf{d}(s)\right\|_{L^{2}(\Omega)}^{2}+\|\mathbf{d}(s)\|_{\mathcal{E}_{1}}^{2}\right) \mathrm{d} s+\frac{1}{2}\|\mathbf{d}(t)\|_{\mathcal{E}_{1}}^{2} .
\end{aligned}
$$


By setting:

$$
z(t)=|\dot{\theta}(t)|^{2}+\left\|\partial_{t} \mathbf{d}(t)\right\|_{L^{2}(\Omega)}^{2}+\frac{1}{2}\|\mathbf{d}(t)\|_{\mathcal{E}_{1}}^{2},
$$

we have:

$$
z(t) \leq \int_{0}^{t} C(s) z(s) \mathrm{d} s .
$$

with $C(\cdot) \in L^{2}(0, T)$. By the Gronwall lemma, we obtain $z(t) \equiv 0$ on $(0, T)$ (because $\theta(0)=0$, $\dot{\theta}(0)=0, \mathbf{d}(\cdot, 0) \equiv 0$ and $\left.\partial_{t} \mathbf{d}(\cdot, 0) \equiv 0\right)$, so that, in the case where $\partial_{t} \mathbf{d} \in L^{\infty}\left(0, T ; \mathcal{E}_{1}\right)$, we have $\theta_{1} \equiv \theta_{2}$ and $\mathbf{d}_{1} \equiv \mathbf{d}_{2}$.

\section{Step 2. Uniqueness of the solution.}

We now adapt the previous proof to the case where $\partial_{t} \mathbf{d}$ is no more in $L^{\infty}\left(0, T ; \mathcal{E}_{1}\right)$, but only in $L^{\infty}\left(0, T ; L^{2}(\Omega)\right)$, and we give the main differences compared to the regular case. To do so, we keep the previous notations and we set $\mathbf{d}_{N}=\Pi_{N}^{0}(\mathbf{d})$ the orthogonal projection ${ }^{3}$ from $L^{2}(\Omega)$ onto $\mathcal{E}^{N}$ of the difference $\mathbf{d}=\mathbf{d}_{2}-\mathbf{d}_{1}$. Thus $\mathbf{d}_{N}$ and especially $\partial_{t} \mathbf{d}_{N}$ are in $L^{\infty}\left(0, T ; \mathcal{E}_{1}\right)$, and it is licit to take $\mathbf{b}=\partial_{t} \mathbf{d}_{N}$ as a test-function in (54). Doing so we obtain:

$$
\begin{aligned}
\rho_{S}\left(\int_{\Omega} \partial_{t t} \mathbf{d} \cdot \partial_{t} \mathbf{d}_{N}+2 \dot{\theta} \int_{\Omega} \partial_{t} \mathbf{d}_{1} \wedge \partial_{t} \mathbf{d}_{N}+2 \dot{\theta}_{2} \int_{\Omega} \partial_{t} \mathbf{d} \wedge \partial_{t} \mathbf{d}_{N}+\ddot{\theta} \int_{\Omega} \mathbf{d}_{1} \wedge \partial_{t} \mathbf{d}_{N}\right. \\
\left.+\ddot{\theta}_{2} \int_{\Omega} \mathbf{d} \wedge \partial_{t} \mathbf{d}_{N}+\mathcal{T}\left(\dot{\theta}_{1}, \mathbf{d}_{1}, \partial_{t} \mathbf{d}_{N}\right)-\mathcal{T}\left(\dot{\theta}_{2}, \mathbf{d}_{2}, \partial_{t} \mathbf{d}_{N}\right)\right)+\left(\left(\mathbf{d}, \partial_{t} \mathbf{d}_{N}\right)\right) \\
\quad=\int_{\Omega} \mathbf{f} \cdot\left(R_{2}-R_{1}\right) \partial_{t} \mathbf{d}_{N}+\int_{\partial \Omega} \mathbf{g} \cdot\left(R_{2}-R_{1}\right) \partial_{t} \mathbf{d}_{N} .
\end{aligned}
$$

As previously we set $y_{N}(t)=\rho_{S}\left\|\dot{\theta} \overrightarrow{e_{z}} \wedge R_{1}\left(\overrightarrow{G \xi}+\mathbf{d}_{1}\right)+R_{1} \partial_{t} \mathbf{d}_{N}\right\|_{L^{2}(\Omega)}^{2}$ for almost every $t \in(0, T)$. We notice that $\int_{\Omega} \partial_{t t} \mathbf{d} \cdot \partial_{t} \mathbf{d}_{N}=\int_{\Omega} \partial_{t t} \mathbf{d}_{N} \cdot \partial_{t} \mathbf{d}_{N}$ and $\left(\left(\mathbf{d}, \partial_{t} \mathbf{d}_{N}\right)\right)=\left(\left(\mathbf{d}_{N}, \partial_{t} \mathbf{d}_{N}\right)\right)$ because of the definitions of $\mathbf{d}_{N}$ and $\mathcal{E}^{N}$. Then adding (53) and (66), we find, as in (58):

$$
\frac{1}{2} \frac{d}{d t}\left(y_{N}+\left\|\mid \mathbf{d}_{N}\right\|^{2}\right)=\mathcal{F}^{N}-\mathcal{R}^{N}
$$

with $\mathcal{F}^{N}=\mathcal{F}_{\mathbf{f}}^{N}+\mathcal{F}_{\mathbf{g}}^{N}$ :

$$
\begin{aligned}
\mathcal{F}_{\mathbf{f}}^{N} & =\dot{\theta} \int_{\Omega}\left[\left(R_{2}-R_{1}\right)\left(\overrightarrow{G \xi}+\mathbf{d}_{2}\right)+R_{1} \mathbf{d}\right] \wedge \mathbf{f}+\int_{\Omega} \mathbf{f} \cdot\left(R_{2}-R_{1}\right) \partial_{t} \mathbf{d}_{N} \\
\mathcal{F}_{\mathbf{g}}^{N} & =\dot{\theta} \int_{\partial \Omega}\left[\left(R_{2}-R_{1}\right)\left(\overrightarrow{G \xi}+\mathbf{d}_{2}\right)+R_{1} \mathbf{d}\right] \wedge \mathbf{g}+\int_{\partial \Omega} \mathbf{g} \cdot\left(R_{2}-R_{1}\right) \partial_{t} \mathbf{d}_{N},
\end{aligned}
$$

and where $\mathcal{R}^{N}$ is given by:

$$
\begin{gathered}
\mathcal{R}^{N}=\rho_{S} \dot{\theta} \int_{\Omega} \mathbf{d}_{1} \wedge\left(\partial_{t t} \mathbf{d}-\partial_{t t} \mathbf{d}_{N}\right)+(\dot{\theta})^{2} \frac{\dot{J}_{1}}{2}+\dot{\theta} \ddot{\theta}_{2}\left(J_{2}-J_{1}\right)+\dot{\theta} \dot{\theta}_{2}\left(\dot{J}_{2}-\dot{J}_{1}\right)+\rho_{S} \dot{\theta} \int_{\Omega} \mathbf{d} \wedge \partial_{t t} \mathbf{d}_{2} \\
+\rho_{S} \dot{\theta} \int_{\Omega} \partial_{t} \mathbf{d}_{1} \wedge \partial_{t} \mathbf{d}_{N}+\rho_{S} \ddot{\theta}_{2} \int_{\Omega} \mathbf{d} \wedge \partial_{t} \mathbf{d}_{N}+2 \rho_{S} \dot{\theta}_{2} \int_{\Omega} \partial_{t} \mathbf{d} \wedge \partial_{t} \mathbf{d}_{N} \\
\quad-\rho_{S} \dot{\theta} \int_{\Omega}\left[\dot{\theta}_{1}\left(\overrightarrow{G \xi}+\mathbf{d}_{1}\right)+\dot{\theta}_{2}\left(\overrightarrow{G \xi}+\mathbf{d}_{2}\right)\right] \cdot \partial_{t} \mathbf{d}_{N}-\rho_{S} \dot{\theta}_{1} \dot{\theta}_{2} \int_{\Omega} \mathbf{d} \cdot \partial_{t} \mathbf{d}_{N} .
\end{gathered}
$$

Most of the terms in $\mathcal{R}^{N}$ are the ones appearing in $\mathcal{R}$ with, in some cases, $\mathbf{d}$ replaced by $\mathbf{d}_{N}$. There are two main differences beetween $\mathcal{R}^{N}$ and $\mathcal{R}$. The first additional term $\rho_{S} \dot{\theta} \int_{\Omega} \mathbf{d}_{1} \wedge\left(\partial_{t t} \mathbf{d}-\right.$

\footnotetext{
${ }^{3}$ There is no confusion possible between $\mathbf{d}_{1}, \mathbf{d}_{2}$ and $\mathbf{d}_{N}$ if we assume $N \geq 3$ (which is reasonable since we want to pass to the limit $N \longrightarrow \infty$ ).
} 
$\partial_{t t} \mathbf{d}_{N}$ ) comes from (53) and from $\dot{y}_{N} / 2$ due to the definition of $y_{N}$. The second extra term is $2 \rho_{S} \dot{\theta}_{2} \int_{\Omega} \partial_{t} \mathbf{d} \wedge \partial_{t} \mathbf{d}_{N}$. We shall prove that these terms go to zero as $N$ goes to infinity.

- Estimate of $\mathcal{R}^{N}$

We now recall the estimates of the different terms of $\mathcal{R}^{N}$. These upper bounds are obtained just as in the regular case :

$$
\begin{gathered}
\left|(\dot{\theta})^{2} \frac{\dot{J}_{1}}{2}\right| \leq C|\dot{\theta}|^{2}, \quad\left|\ddot{\theta}_{2}\left(J_{2}-J_{1}\right)\right| \leq C(t) \mid \dot{\theta}\|\mathbf{d}\| \|_{L^{2}(\Omega)}, \\
\left|\dot{\theta} \int_{\Omega} \partial_{t} \mathbf{d}_{1} \wedge \partial_{t} \mathbf{d}_{N}\right| \leq C|\dot{\theta}|\left\|\partial_{t} \mathbf{d}_{N}\right\|_{L^{2}(\Omega)}, \quad\left|\ddot{\theta}_{2} \int_{\Omega} \mathbf{d} \wedge \partial_{t} \mathbf{d}_{N}\right| \leq\left. C(t)\|\mathbf{d}\|\right|_{L^{2}(\Omega)}\left\|\partial_{t} \mathbf{d}_{N}\right\|_{L^{2}(\Omega)}, \\
\left|\dot{\theta} \int_{\Omega}\left[\dot{\theta}_{1}\left(\overrightarrow{G \xi}+\mathbf{d}_{1}\right)+\dot{\theta}_{2}\left(\overrightarrow{G \xi}+\mathbf{d}_{2}\right)\right] \cdot \partial_{t} \mathbf{d}_{N}\right| \leq C|\dot{\theta}|\left\|\partial_{t} \mathbf{d}_{N}\right\|_{L^{2}(\Omega)}, \\
\left|\dot{\theta}_{1} \dot{\theta}_{2} \int_{\Omega} \mathbf{d} \cdot \partial_{t} \mathbf{d}_{N}\right| \leq C|| \mathbf{d}\left\|_{L^{2}(\Omega)}\right\| \partial_{t} \mathbf{d}_{N} \|_{L^{2}(\Omega)} .
\end{gathered}
$$

We now treat the terms of $\mathcal{R}^{N}$ that need sharper estimates:

$$
\begin{aligned}
\left|\dot{\theta} \int_{\Omega} \mathbf{d}_{1} \wedge\left(\partial_{t t} \mathbf{d}-\partial_{t t} \mathbf{d}_{N}\right)\right| & \leq\left. C|\dot{\theta}|\left\|\partial_{t t} \mathbf{d}-\partial_{t t} \mathbf{d}_{N}\right\|\right|_{\left(H^{1}(\Omega)\right)^{\prime}} \\
\left|\dot{\theta}_{2}\left(\dot{J}_{2}-\dot{J}_{1}\right)\right| & =2 \rho_{S}|\dot{\theta}|\left|\dot{\theta}_{2}\right|\left|\int_{\Omega} \mathbf{d} \cdot \partial_{t} \mathbf{d}_{2}+\int_{\Omega}\left(\overrightarrow{G \xi}+\mathbf{d}_{1}\right) \cdot\left(\partial_{t} \mathbf{d}_{N}-\partial_{t} \mathbf{d}_{N}+\partial_{t} \mathbf{d}\right)\right| \\
& \leq C|\dot{\theta}|\left(\|\mathbf{d}\|_{L^{2}(\Omega)}+\left\|\partial_{t} \mathbf{d}_{N}\right\|_{L^{2}(\Omega)}+\left\|\partial_{t} \mathbf{d}-\partial_{t} \mathbf{d}_{N}\right\|_{L^{2}(\Omega)}\right), \\
\left|\dot{\theta} \int_{\Omega} \mathbf{d} \wedge \partial_{t t} \mathbf{d}_{2}\right| & \leq C(t)|\dot{\theta}|\left(\left|\left\|\mathbf{d}-\mathbf{d}_{N}\right\|\right|+\left\||| \mathbf{d}_{N}\right\| \mid\right), \\
\left|\dot{\theta}_{2} \int_{\Omega} \partial_{t} \mathbf{d} \wedge \partial_{t} \mathbf{d}_{N}\right| & =\left|\dot{\theta}_{2} \int_{\Omega}\left(\partial_{t} \mathbf{d}-\partial_{t} \mathbf{d}_{N}\right) \wedge \partial_{t} \mathbf{d}_{N}\right| \\
& \leq C\left\|\partial_{t} \mathbf{d}-\partial_{t} \mathbf{d}_{N}\right\|_{L^{2}(\Omega)}\left\|\partial_{t} \mathbf{d}_{N}\right\|_{L^{2}(\Omega)},
\end{aligned}
$$

with $C(\cdot) \in L^{2}(0, T)$.

- Estimates of $\mathcal{F}^{N}$.

For the term $\mathcal{F}_{\mathbf{f}}^{N}$ we have directly:

$$
\left|\mathcal{F}_{\mathbf{f}}^{N}\right| \leq C(t)\left(|\dot{\theta}||\theta|+|\dot{\theta}|\left|\mathbf{d}\left\|_{L^{2}(\Omega)}+|\theta|\right\| \partial_{t} \mathbf{d}_{N} \|_{L^{2}(\Omega)}\right)\right.
$$

with $C(\cdot) \in L^{2}(0, T)$. We next have to treat $\mathcal{F}_{\mathbf{g}}^{N}$. First we have

$$
\begin{gathered}
\left|\dot{\theta} \int_{\partial \Omega}\left[\left(R_{2}-R_{1}\right)\left(\overrightarrow{G \xi}+\mathbf{d}_{2}\right)\right] \wedge \mathbf{g}\right| \leq C|\theta||\dot{\theta}|, \\
\int_{\partial \Omega} \mathbf{g} \cdot\left(R_{2}-R_{1}\right) \partial_{t} \mathbf{d}_{N} \leq \frac{d}{d t}\left(\int_{\partial \Omega} \mathbf{g} \cdot\left(R_{2}-R_{1}\right) \mathbf{d}_{N}\right)+C(t)(|\dot{\theta}|+|\theta|)||\left|\mathbf{d}_{N}\right| \|,
\end{gathered}
$$

and

$$
\begin{aligned}
\left|\dot{\theta} \int_{\partial \Omega} R_{1} \mathbf{d} \wedge \mathbf{g}\right| & \leq|\dot{\theta}|\left(\left|\int_{\partial \Omega} R_{1}\left(\mathbf{d}-\mathbf{d}_{N}\right) \wedge \mathbf{g}\right|+\left|\int_{\partial \Omega} R_{1} \mathbf{d}_{N} \wedge \mathbf{g}\right|\right) \\
& \leq C|\dot{\theta}|\left(||\left|\mathbf{d}-\mathbf{d}_{N}\right|||+||\left|\mathbf{d}_{N}\right|||\right) .
\end{aligned}
$$

Summing up all these estimates, we arrive at:

$$
\begin{aligned}
\frac{1}{2} \frac{d}{d t}\left(y_{N}+\left\|\left|\mathbf{d}_{N} \|\right|^{2}\right) \leq\right. & C(t)\left(|\theta|^{2}+|\dot{\theta}|^{2}+\left\|\left|\mathbf{d}_{N}\left\|\left.\right|^{2}+\right\| \partial_{t} \mathbf{d}_{N} \|_{L^{2}(\Omega)}^{2}\right.\right.\right. \\
& +\|\mathbf{d}\|_{L^{2}(\Omega)}^{2}+\left\|\partial_{t} \mathbf{d}-\partial_{t} \mathbf{d}_{N}\right\|_{L^{2}(\Omega)}^{2}+\left\|\partial_{t t} \mathbf{d}-\partial_{t t} \mathbf{d}_{N}\right\|_{\left(H^{1}(\Omega)\right)^{\prime}}^{2} \\
& \left.+\left\|\left|\mathbf{d}-\mathbf{d}_{N}\right|\right\|^{2}\right)+\frac{d}{d t}\left(\int_{\partial \Omega} \mathbf{g} \cdot\left(R_{2}-R_{1}\right) \mathbf{d}_{N}\right) .
\end{aligned}
$$


From the estimates of the terms of $\mathcal{R}^{N}$ and in the terms of $\mathcal{F}^{N}$ we obtain easily an estimate analogous to $(65)$ :

$$
\begin{aligned}
|\dot{\theta}(t)|^{2}+\left\|\partial_{t} \mathbf{d}_{N}(t)\right\|_{L^{2}(\Omega)}^{2}+\left\|\mathbf{d}_{N}(t)\right\|_{\mathcal{E}_{1}}^{2} & \\
& \leq \int_{0}^{t} C(s)\left(|\dot{\theta}(s)|^{2}+\left\|\partial_{t} \mathbf{d}_{N}(s)\right\|_{L^{2}(\Omega)}^{2}+\left\|\mathbf{d}_{N}(s)\right\|_{L^{\infty}\left(\mathcal{E}_{1}\right)}^{2}\right) \mathrm{d} s+w_{N}(t)
\end{aligned}
$$

where:

$$
\begin{aligned}
w_{N}(t)=C_{0} \int_{0}^{t} C(s)\left(\|\|\left(\mathbf{d}-\mathbf{d}_{N}\right)(s)\left\|\left.\right|^{2}+\right\|\left(\partial_{t} \mathbf{d}-\partial_{t} \mathbf{d}_{N}\right)(s) \|_{L^{2}(\Omega)}^{2}\right. & \\
& \left.+\left\|\left(\partial_{t t} \mathbf{d}-\partial_{t t} \mathbf{d}_{N}\right)(s)\right\|_{\left(H^{1}(\Omega)\right)^{\prime}}^{2}\right) \mathrm{d} s .
\end{aligned}
$$

We set:

$$
z_{N}(t)=|\dot{\theta}(t)|^{2}+\left\|\partial_{t} \mathbf{d}_{N}(t)\right\|_{L^{2}(\Omega)}^{2}+\left\|\mathbf{d}_{N}(t)\right\|_{\mathcal{E}_{1}}^{2},
$$

thus

$$
z_{N}(t) \leq \int_{0}^{t} C(s) z(s) \mathrm{d} s+w_{N}(t) .
$$

Then, by the Gronwall lemma, we obtain:

$$
z_{N}(t) \leq w_{N}(t) \exp \left(\int_{0}^{t} C(s) \mathrm{d} s\right) .
$$

But, as $\mathbf{d}-\mathbf{d}_{N}$ goes to zero strongly in $H^{2}\left(0, T ;\left(H^{1}(\Omega)\right)^{\prime}\right) \cap H^{1}\left(0, T ; L^{2}(\Omega)\right) \cap L^{2}\left(0, T ; \mathcal{E}_{1}\right)$, we have $\lim _{N \longrightarrow \infty} w_{N}(t)=0$ for almost every $t \in(0, T)$. Then $\lim _{N \longrightarrow \infty} z_{N}(t)=0$ implies $\theta \equiv 0$ (because $\theta(0)=0)$ and:

$$
\lim _{N \longrightarrow \infty}\left\|\mathbf{d}_{N}\right\|_{L^{\infty}\left(0, t ; \mathcal{E}_{1}\right)}^{2}=\lim _{N \longrightarrow \infty}\left\|\partial_{t} \mathbf{d}_{N}\right\|_{L^{\infty}\left(0, t ; L^{2}(\Omega)\right)}^{2}=0,
$$

which implies $\mathbf{d} \equiv 0$. This ends the proof of the uniqueness of the rotation angle and of the elastic displacement.

\subsubsection{Existence and uniqueness of a maximal solution}

We have thus proven that there exists a time $T>0$ such that on the interval $(0, T)$ the solution of (30) exists and is unique, under the condition $\mathbf{d} \in C^{0}\left([0, T] ; \mathbf{Y}_{0}^{\delta}\right)$ where $\delta$ is given such that $0<\delta<\int_{\Omega}\left(\overrightarrow{G \xi}+\mathbf{d}_{0}\right) \cdot \overrightarrow{G \xi}$. By a standard argument there exists a unique maximal solution on $\left(0, T^{*}\right)$. Next we verify that

$$
\begin{aligned}
& -T^{*}=+\infty \text { or } \\
& -\lim _{t \longrightarrow T^{*}} \int_{\Omega}(\overrightarrow{G \xi}+\mathbf{d}) \cdot \overrightarrow{G \xi}=0 .
\end{aligned}
$$

Indeed thanks to the regularities of the solution and to the energy estimates satisfied by the solution, we can extend it untill $\int_{\Omega}(\overrightarrow{G \xi}+\mathbf{d}) \cdot \overrightarrow{G \xi}>0$. That concludes the proof of Theorem 1 .

\subsection{Proof of Theoreme 2}

In this subsection we prove that Theorem 1 is equivalent to Theorem 2. Starting from the system (30) satisfied by the translation the rotation and the elastic displacement we build a global displacement and the system satisfied by it. We consider:

$$
(\boldsymbol{\tau}, \theta, \mathbf{d}) \in H^{2}(0, T) \times H^{2}(0, T) \times H^{2}\left(0, T ;\left(H^{1}(\Omega)\right)^{\prime}\right) \cap W^{1, \infty}\left(0, T ; L^{2}(\Omega)\right) \cap L^{\infty}\left(0, T ; H^{1}(\Omega)\right)
$$


the unique solution of (30). Then thanks to the subsection 2.1, we can associate $\phi$ such that $\forall t \in[0, T]:$

$$
\phi(\cdot, t)=\boldsymbol{\tau}(t)+R_{\theta(t)}(\overrightarrow{G \xi}+\mathbf{d}(\cdot, t)) \in \mathbf{X}_{0} \quad \text { with } \quad R_{\theta(t)}=R=\left(\begin{array}{cc}
\cos \theta(t) & -\sin \theta(t) \\
\sin \theta(t) & \cos \theta(t)
\end{array}\right) .
$$

We define $\mathbf{u}=\phi-\operatorname{Id}_{\mathbb{R}^{2}}$ that belongs to $H^{2}\left(0, T ;\left(H^{1}(\Omega)\right)^{\prime}\right) \cap W^{1, \infty}\left(0, T ; L^{2}(\Omega)\right) \cap L^{\infty}\left(0, T ; H^{1}(\Omega)\right)$.

- Considering the first equation of (30), we have for all $\overline{\boldsymbol{\tau}} \in H^{1}(0, T)$ :

$$
m \ddot{\boldsymbol{\tau}} \cdot \overline{\boldsymbol{\tau}}=\rho_{S} \frac{d}{d t}\left(\int_{\Omega} \partial_{t} \mathbf{u} \cdot \overline{\boldsymbol{\tau}}\right)-\rho_{s} \int_{\Omega} \mathbf{u} \cdot \dot{\overline{\boldsymbol{\tau}}}=\int_{\Omega} \mathbf{f} \cdot \overline{\boldsymbol{\tau}}+\int_{\partial \Omega} \mathbf{g} \cdot \overline{\boldsymbol{\tau}} \quad \text { in } \mathcal{D}^{\prime}(0, T)
$$

since $\mathbf{d} \in C^{0}\left([0, T] ; \mathbf{Y}_{0}\right)$ and $\partial_{t} \mathbf{d} \in L^{\infty}\left(0, T ; \mathcal{E}_{0}\right)$.

- We consider now the second equation of (30). We remark that

$$
\begin{array}{rlrl}
\frac{1}{\rho_{S}}\left(J \dot{\theta} \overrightarrow{e_{z}}\right. & \left.+\rho_{S} \int_{\Omega} \mathbf{d} \wedge \partial_{t} \mathbf{d}\right)=\dot{\theta} \int_{\Omega}(\overrightarrow{G \xi}+\mathbf{d})^{2} \overrightarrow{e_{z}}+R \int_{\Omega} \mathbf{d} \wedge \partial_{t} \mathbf{d} & & \\
=\int_{\Omega} R(\overrightarrow{G \xi}+\mathbf{d}) & \wedge\left[\dot{\theta} \overrightarrow{e_{z}} \wedge R(\overrightarrow{G \xi}+\mathbf{d})\right]+R \int_{\Omega}(\overrightarrow{G \xi}+\mathbf{d}) \wedge \partial_{t} \mathbf{d} & \text { because } \partial_{t} \mathbf{d} \in L^{\infty}\left(0, T ; \mathcal{E}_{0}\right) \\
=\int_{\Omega} R(\overrightarrow{G \xi}+\mathbf{d}) \wedge\left[\dot{\boldsymbol{\tau}}+\dot{\theta} \overrightarrow{e_{z}} \wedge R(\overrightarrow{G \xi}+\mathbf{d})+R \partial_{t} \mathbf{d}\right] & \text { because } \mathbf{d} \in L^{\infty}\left(0, T ; \mathcal{E}_{0}\right) .
\end{array}
$$

Then since $\mathbf{u}=\boldsymbol{\tau}+R(\overrightarrow{G \xi}+\mathbf{d})-\overrightarrow{O \xi}$ and $\dot{R}=\dot{\theta} \overrightarrow{e_{z}} \wedge R$, we obtain after multiplying the second equation of (30), by $\bar{\theta} \in H^{1}(0, T)$ :

$$
\begin{aligned}
\rho_{S} \frac{d}{d t}\left(\int _ { \Omega } \left[\bar{\theta} \overrightarrow{e_{z}} \wedge\right.\right. & \left.R(\overrightarrow{G \xi}+\mathbf{d})] \cdot \partial_{t} \mathbf{u}\right)-\rho_{S}\left(\int_{\Omega}\left[\dot{\bar{\theta}} \overrightarrow{e_{z}} \wedge R(\overrightarrow{G \xi}+\mathbf{d})\right] \cdot \mathbf{u}\right) \\
& =\int_{\Omega} \mathbf{f} \cdot\left[\bar{\theta} \overrightarrow{e_{z}} \wedge R(\overrightarrow{G \xi}+\mathbf{d})\right]+\int_{\partial \Omega} \mathbf{g} \cdot\left[\bar{\theta} \overrightarrow{e_{z}} \wedge R(\overrightarrow{G \xi}+\mathbf{d})\right] \text { in } \mathcal{D}^{\prime}(0, T) .
\end{aligned}
$$

- We now look at the last equation of (30). We have for all $\mathbf{b} \in H^{1}\left(0, T ; \mathcal{E}_{1}\right)$ :

$$
\int_{\Omega} \partial_{t} \mathbf{u} \cdot R \mathbf{b}=\int_{\Omega}\left(\dot{\theta} \overrightarrow{e_{z}} \wedge R(\overrightarrow{G \xi}+\mathbf{d})+R \partial_{t} \mathbf{d}\right) \cdot R \mathbf{b}=\dot{\theta} \overrightarrow{e_{z}} \cdot \int_{\Omega} \mathbf{d} \wedge \mathbf{b}+\int_{\Omega} \partial_{t} \mathbf{d} \cdot \mathbf{b},
$$

and

$$
\frac{d}{d t}\left(\int_{\Omega} \partial_{t} \mathbf{u} \cdot R \mathbf{b}\right)=\ddot{\theta} \overrightarrow{e_{z}} \cdot \int_{\Omega} \mathbf{d} \wedge \mathbf{b}+\dot{\theta} \overrightarrow{e_{z}} \cdot \int_{\Omega} \partial_{t} \mathbf{d} \wedge \mathbf{b}+\int_{\Omega} \partial_{t t} \mathbf{d} \cdot \mathbf{b}+\int_{\Omega} \partial_{t} \mathbf{u} \cdot R \partial_{t} \mathbf{b} .
$$

Moreover, we have easily:

$$
\int_{\Omega} \partial_{t} \mathbf{u} \cdot \dot{R} \mathbf{b}=\int_{\Omega}\left[\dot{\theta} \overrightarrow{e_{z}} \wedge R(\overrightarrow{G \xi}+\mathbf{d})+R \partial_{t} \mathbf{d}\right] \cdot\left(\dot{\theta} \overrightarrow{e_{z}} \wedge R \mathbf{b}\right)=(\dot{\theta})^{2} \int_{\Omega}(\overrightarrow{G \xi}+\mathbf{d}) \cdot \mathbf{b}-\dot{\theta} \int_{\Omega} \partial_{t} \mathbf{d} \wedge \mathbf{b}
$$

Consequently we deduce that (30) could be written, for all $\mathbf{b} \in H^{1}\left(0, T ; \mathcal{E}_{1}\right)$ :

$$
\rho_{S} \frac{d}{d t}\left(\int_{\Omega} \partial_{t} \mathbf{u} \cdot R \mathbf{b}\right)-\int_{\Omega} \partial_{t} \mathbf{u} \cdot R \partial_{t} \mathbf{b}-\rho_{S} \int_{\Omega} \partial_{t} \mathbf{u} \cdot \dot{R} \mathbf{b}+\int_{\Omega} \bar{\sigma}(\mathbf{d}): \nabla \mathbf{b}=\int_{\Omega} \mathbf{f} \cdot R \mathbf{b}+\int_{\partial \Omega} \mathbf{g} \cdot R \mathbf{b} .
$$

- Finally let $\mathbf{v} \in H^{1}(\Omega)$, then there exists a unique triplet $(\overline{\boldsymbol{\tau}}, \bar{\theta}, \mathbf{b}) \in \mathbb{R}^{2} \times W^{1, \infty}(0, T) \times$ $W^{1, \infty}\left(0, T ; \mathcal{E}_{1}\right)$ such that $\mathbf{v}=\overline{\boldsymbol{\tau}}+\bar{\theta} \overrightarrow{e_{z}} \wedge R(\overrightarrow{G \xi}+\mathbf{d})+R \mathbf{b}$. Summing up (68)-(69)-(70), we obtain:

$$
\rho_{S} \frac{d}{d t}\left(\int_{\Omega} \partial_{t} \mathbf{u} \cdot \mathbf{v}\right)-\rho_{S} \int_{\Omega} \partial_{t} \mathbf{u} \cdot \partial_{t} \mathbf{v}+\int_{\Omega} \bar{\sigma}(\mathbf{d}): \nabla \mathbf{b}=\int_{\Omega} \mathbf{f} \cdot \mathbf{v}+\int_{\partial \Omega} \mathbf{g} \cdot \mathbf{v}
$$


since $\partial_{t} \mathbf{v}=\dot{\bar{\theta}} \overrightarrow{e_{z}} \wedge R(\overrightarrow{G \xi}+\mathbf{d})+R \partial_{t} \mathbf{b}+\bar{\theta} \overrightarrow{e_{z}} \wedge \partial_{t}[R(\overrightarrow{G \xi}+\mathbf{d})]+\dot{R} \mathbf{b}$ and using the fact that:

$$
\int_{\Omega} \partial_{t} \mathbf{u} \cdot\left(\overrightarrow{e_{z}} \wedge \partial_{t}[R(\overrightarrow{G \xi}+\mathbf{d})]\right)=\overrightarrow{e_{z}} \cdot \int_{\Omega} \partial_{t}[R(\overrightarrow{G \xi}+\mathbf{d})] \wedge \partial_{t}[\boldsymbol{\tau}+R(\overrightarrow{G \xi}+\mathbf{d})-\overrightarrow{O \xi}]=0 .
$$

Then the weak formulation satisfied by $\mathbf{u}$ is the following:

$$
\forall \mathbf{v} \in H^{1}(\Omega), \quad \rho_{S} \frac{d}{d t}\left(\int_{\Omega} \partial_{t} \mathbf{u} \cdot \mathbf{v}\right)+\int_{\Omega} \bar{\sigma}(\mathbf{d}): \nabla \mathbf{b}=\int_{\Omega} \mathbf{f} \cdot \mathbf{v}+\int_{\partial \Omega} \mathbf{g} \cdot \mathbf{v} \quad \text { in } \quad \mathcal{D}^{\prime}(0, T)
$$

with $\mathbf{b}$ associated with $\mathbf{v}$ through (9). Moreover the initial conditions are satisfied:



Thus any solution of (30) is a solution of (31). The reciprocal proposition is also true. Thus Theoreme 1 and Theoreme 2 are equivalent.

\section{Conclusion}

We have obtained a wellposed system that models the motion of a bidimensional body in large displacement-small strain. The same kind of model can be obtained for the 3D case (see [7], [8]). For the 3D case the same uniqueness and existence result can be proved.

\section{References}

[1] V.I. Arnold. Mathematical methods of classical mechanics, translated from the Russian by K. Vogtmann and A. Weinstein, volume 60 of Graduate Texts in Mathematics. Springer-Verlag, New York, 2nd edition, 1989.

[2] P. G. Ciarlet. Mathematical elasticity. Volume I. North-Holland Publishing Co., Amsterdam, 1988. Three-dimensional elasticity.

[3] E. A. Coddington and N. Levinson. Theory of Ordinary Differential Equations. McGraw Hill, New York, 1955.

[4] C. Cohen-Tannoudji, B. Diu, and F. Laloë. Mécanique quantique, Volume 2. Hermann, Paris, 1973.

[5] G. Duvaut and J.-L. Lions. Les inéquations en mécanique et en physique. Dunod, Paris, 1972. Travaux et Recherche Mathématique, N.21.

[6] C. Farhat, K. Pierson, and M. Lesoinne. The second generation of FETI methods and their application to the parallel solution of large-scale linear and geometrically nonlinear structural analysis problems. Computer Methods in Applied Mechanics and Engineering, 184:333-374, 2000.

[7] B. Fraeijs De Veubeke. The dynamics of flexible bodies. Int. J. Engng Sci., 14:895-913, 1976.

[8] C. Grandmont and Y. Maday. Some remarks on fluid-structure interaction problems in case of rigid body plus small perturbations. CFSWA proceedings, Springer series "Notes on numerical fluid mechanics", 2003.

[9] C. Grandmont, Y. Maday, and P. Métier. Existence of a solution for an unsteady elasticity problem in large displacement and small perturbation. C. R. Acad. Sci. Paris Sér. I Math., 334:521-526, 2002. 
[10] J.L. Lions. Quelques méthodes de résolution des problèmes aux limites non linéaires. Dunod, Gauthier-Villars, Paris, 1969.

[11] J.L. Lions and E. Magenes. Problèmes aux limites non homogènes et applications., volume 1. Dunod, Paris, 1968. 